\title{
Fluctuations in fluids in thermal nonequilibrium states below the convective Rayleigh-Bénard instability
}

\author{
José M. Ortiz de Zárate ${ }^{1}$ \\ Departamento de Física Aplicada I, Facultad de Ciencias Físicas, Universidad \\ Complutense, E28040 Madrid, Spain \\ Jan V. Sengers \\ Institute for Physical Science and Technology and Department of Chemical \\ Engineering, University of Maryland, College Park, MD 20742, USA
}

\begin{abstract}
Starting from the linearized fluctuating Boussinesq equations we derive an expression for the structure factor of fluids in stationary convection-free thermal nonequilibrium states, taking into account both gravity and finite-size effects. It is demonstrated how the combined effects of gravity and finite size causes the structure factor to go through a maximum value as a function of the wave number $q$. The appearance of this maximum is associated with a crossover from a $q^{-4}$ dependence for larger $q$ to a $q^{2}$ dependence for very small $q$. The relevance of this theoretical result for the interpretation of light scattering and shadowgraph experiments is elucidated. The relationship with studies on various aspects of the problem by other investigators is discussed. The paper thus provides a unified treatment for dealing with fluctuations in fluid layers subjected to a stationary temperature gradient regardless of the sign of the Rayleigh number $R$, provided that $R$ is smaller than the critical value $R_{\mathrm{c}}$ associated with the appearance of Rayleigh-Bénard convection.
\end{abstract}

Key words: Boussinesq equations, Light scattering, Nonequilibrium fluctuations, Rayleigh-Bénard convection, Shadowgraph experiments, Swift-Hohenberg equation PACS: 05.40, 05.70.L, 44.25, 47.20, 78.35

$\overline{1 \text { Corresponding author }}$

Preprint submitted to Elsevier Preprint 23 July 2013 


\section{Introduction}

Questions concerning the nature of thermal fluctuations in fluids in thermal nonequilibrium states have been the subject of many studies during the past two decades. Specifically, in this paper we consider thermal fluctuations in a horizontal layer of a one-component fluid that is heated either from above or from below in such a way that the fluid is still in a stable convection-free state. A number of different and independent approaches for dealing with various aspects of this problem have appeared in the literature.

Most of the earlier studies focused on the nonequilibrium fluctuations close to the convective Rayleigh-Bénard instability because of their possible influence on the pattern-selection process when convection appears [1-4]. The effects of both linear and nonlinear terms in the hydrodynamic fluctuations close to the convection threshold have been evaluated. This line of research turned out to be very important, mainly because it lead to the development of convection model equations for dealing with spatiotemporal-pattern formation above the threshold, as reviewed by Cross and Hohenberg [5].

A second and independent line of research developed when investigators became interested in the nature of the fluctuations in nonequilibrium steady states far away from any hydrodynamic instability. The first important theoretical work along this line was done by Kirkpatrick et al., who calculated the correct expression for the structure factor of a fluid subjected to a stationary temperature gradient [6]. Althought their result was originally obtained on the basis of the same mode coupling theory that in equilibrium leads to long-time tail contributions to the correlation functions for the transport coefficients, it turned out that the same result could also be obtained from the simpler scheme of Landau's fluctuating hydrodynamics [7-9]. This line of research received an important momentum from the experimental side when the research group of Sengers and coworkers verified from light-scattering experiments the main conclusion of the theoretical result, namely that the nonequilibrium contribution to the structure factor of a liquid is proportional to the square of the temperature gradient $\nabla T$ and inversely proportional to the fourth power of the wave number $q$ of the fluctuations [10-12]. Theory and experiments have subsequently been extended to also deal with nonequilibrium fluctuations in liquid mixtures and polymer solutions, as reviewed elsewhere [13].

A third line of independent research has focused on the real-space behavior of the nonequilibrium correlations resulting from the heat-diffusion equation [1417]. A study of the correlations resulting from the heat-diffusion equation is equivalent to a study of the nonequilibrium correlations in a liquid in the direction co-incident with the temperature gradient. This line of research has shown how the nonequilibrium correlation function of the fluctuating variables 
encompasses the entire system not involving any intrinsic lenght scales. The real-space analysis has demonstrated the long-range nature of the nonequilibrium fluctuations in the direction of the temperature gradient, which has been further confirmed by numerical integration of the equations [14], by computer simulations [18] and by a lattice-gas automaton approach [19].

It is our present goal to further extend the second aforementioned research so as to describe nonequilibrium fluctuations far away from any convective instability, nonequilibrium fluctuations close to the convective instability and nonequilibrium fluctuations produced by the heat-diffusion equation from one unified point of view. The original work of Kirkpatrick et al. and of others $[6,7,9]$ yielded an expression for the structure factor of a fluid in thermally nonequilibrium states without considering any gravity or boundary effects. Segrè at al. extended the theory to include gravity effects, first in a onecomponent liquid [20] and then in a liquid mixture [21]. They predicted that gravity will cause the singular $q^{-4}$ dependence of the structure factor to saturate at small values of $q$. This prediction was confirmed experimentally by Vailati and Giglio from light-scattering experiments at ultra-small scattering angles $[22,23]$. More recently, Ortiz de Zárate et al. evaluated the nonequilibrium structure factor including finite-size effects but neglecting gravity [24,25]. They concluded that deviations from the $q^{-4}$ dependence due to finite-size effects will be just as important as deviations due to gravity. Hence, for a quantitative interpretation of ultra-low-angle light scattering or shadowgraph experiments it is important to account for both gravity and finite-size effects.

In this paper we extend the previous work so as to include gravity and finitesize effects simultaneously. Starting from the linearized fluctuating Boussinesq equations, we shall derive a complete expression for the structure factor of the nonequilibrium fluid making no other approximations than the ones incorporated in the Boussinesq equations. We shall then show that the resulting expression is capable of describing nonequilibrium fluctuations both far away and close to the convective instability. Close to the convective instability we shall recover the linear Swift-Hohenberg correlation function as an approximation to our more complete result. From the study of the behavior of the nonequilibrium correlation function in real space, we shall recover with appropriate approximations, expressions and plots previously obtained by other investigators for the case of the heat-diffusion equation. Thus it will be shown how these various conclusions can be reached from one single theoretical result.

Attempts to arrive at a unified description of nonequilibrium fluctuations both far away and close to the convective instability has been made by Kirkpatrick and Cohen [26] and by Schmitz and Cohen [27], but the consequences of their theoretical results for the interpretation of actual experiments are not clear. In contrast, we shall be able to make a connection with available experimental data for the thermal fluctuations near the convective Rayleigh-Bénard insta- 
bility [28].

There has been an extensive debate whether fluctuating hydrodynamics can properly account for the thermal fluctuations close to the onset of convection [29-33], but the most recent answer to this question appears to be positive $[28,34]$. The present paper will provide additional evidence for the validity of fluctuating hydrodynamics to describe nonequilibrium fluctuations close to the onset of the classical Rayleigh-Bénard instability in simple fluids.

We shall proceed as follows. We start from the linear fluctuating Boussinesq equations which will be written in a form most suitable for our analysis in Section 2. In Section 3 we review the derivation of the well-known expression for the structure factor of a fluid subjected to a stationary temperature gradient without taking into account the presence of boundaries, but including the effects of gravity. The relationship of the resulting expression for the effect of gravity with that obtained previously by Segrè et al. [20] will be elucidated. In Section 4 we then consider the modifications to the nonequilibrium structure factor due to the finite height of the fluid layer. In Section 5 we present a detailed analysis of how the combined effects of gravity and finite-size will appear in low-angle light scattering and in shadowgraph experiments. Sections 4 and 5 contain the most important new features of the present work. In Section 6 we introduce an approximation valid close to the convective instability and show how one can recover the linear Swift-Hohenberg model for the fluctuations near the convective threshold. In Section 7 we discuss the spatial behavior of the nonequilibrium structure factor and we shall make contact with the results obtained by previous investigators for the long-range nature of the nonequilibrium fluctuations in the direction of the temperature gradient. Our results are summarized in Section 8.

\section{$2 \quad$ Linearized fluctuating Boussinesq equations}

We consider a fluid layer between two horizontal plates separated by a distance $L$. The fluid layer is subjected to a temperature gradient in the vertical direction by maintaining the plates at two different temperatures. The size of the system in the two horizontal $\mathrm{X}$ - and $\mathrm{Y}$-directions is much larger than the size $L$ in the vertical Z-direction.

To determine the structure factor of the fluid we consider small fluctuations around the conductive solution. These small fluctuations shall be described by the linearized Boussinesq equations supplemented with random noise terms, as first considered by Zaitsev and Shliomis and by Swift and Hohenberg for studying the influence of thermal noise close to the convective instability $[1,4]$. Use of the Boussinesq approximation to the full hydrodynamics equations 
implies that we neglect the sound modes and consider only density fluctuations caused by temperature fluctuations. The temperature gradient is applied along the Z-direction and is written in the form:

$$
\nabla T_{0}=\nabla T_{0} \hat{z}
$$

where $T_{0}=\langle T\rangle$ is the average local value of the temperature $T$ and where $\nabla T_{0}=d T_{0} / d z$. Hence, $\nabla T_{0}$ is positive when the fluid layer is heated from above and negative when heated from below. The gravitational force $\mathbf{g}$ is directed in the negative Z-direction:

$$
\mathbf{g}=-g \hat{z}
$$

where $g$ is the gravitational acceleration constant. In this notation, the Rayleigh number may be defined as:

$$
R=\frac{\alpha L^{4} \mathbf{g} \cdot \nabla T_{0}}{\nu D_{T}}=-\frac{\alpha L^{4} g \nabla T_{0}}{\nu D_{T}}
$$

where $\alpha$ is the thermal expansion coefficient, $\nu$ the kinematic viscosity and $D_{T}$ the thermal diffusivity of the fluid.

We shall evaluate the structure factor of the fluid maintained in a convectionfree thermal nonequilibrium state, where the average value $\langle\mathbf{v}\rangle$ of the fluid velocity $\mathbf{v}$ will be zero. Such states correspond to both negative and positive values of the Rayleigh number $R$ as long as $R$ is smaller than the critical value $R_{\mathrm{c}}$. For this purpose we write the linearized fluctuating Boussinesq equations in the form $[4,5]$ :

$$
\begin{gathered}
\frac{\partial}{\partial t}\left(\nabla^{2} w\right)=\nu \nabla^{2}\left(\nabla^{2} w\right)+\alpha g\left(\frac{\partial^{2} \theta}{\partial x^{2}}+\frac{\partial^{2} \theta}{\partial y^{2}}\right)+F_{1} \\
\frac{\partial \theta}{\partial t}=D_{T} \nabla^{2} \theta-w \nabla T_{0}+F_{2}
\end{gathered}
$$

where $\theta=T-T_{0}$ represents the local fluctuating temperature and $w$ is the fluctuating Z-component of the fluid velocity $\mathbf{v}$. To eliminate the stationary pressure gradient from the equations we find it convenient to consider Eq. (4a) for $\nabla^{2} w$, rather than an equation for the fluctuating fluid velocity $\mathbf{v}$ itself [35]. Finally, $F_{1}$ and $F_{2}$ represent the contributions from rapidly varying short-range fluctuations and are related to Landau's random stress tensor $\delta$ T and random heat flow $\delta \mathbf{Q}$ in such a way that [36]:

$$
F_{1}=\frac{1}{\rho}\{\boldsymbol{\nabla} \times[\boldsymbol{\nabla} \times(\boldsymbol{\nabla} \cdot \delta \mathrm{T})]\}_{z}
$$




$$
F_{2}=-\frac{D_{T}}{\lambda} \boldsymbol{\nabla}(\delta \mathbf{Q})
$$

where $\rho$ and $\lambda$ are the density and the thermal conductivity of the fluid, while the subscript $z$ in Eq. (5a) indicates that $F_{1}$ has to be identified with the Z-component of the vector between the curly brackets. We note that we are here considering only the effects of additive noise represented by $F_{1}$ and $F_{2}$ in Eqs. (4). Multiplicative noise has been also considered in the literature [37], but that subject lies outside the scope of the present paper. Since $\langle\theta\rangle=0$ and $\langle w\rangle=0$ everywhere in the fluid layer, we note that the solution of the fluctuating Boussinesq equations will be independent of the sign adopted for $\theta$ or $w[4,5,35,36]$.

The conditions for the validity of the Boussinesq equations, also referred to as Oberbeck-Boussinesq equations, have been discussed extensively in the literature [38-40]. Specifically, in deriving the Boussinesq equations from the more general hydrodynamic equations, one neglects the temperature dependence of the various thermophysical properties, except for the density which is taken to vary linearly with temperature. Deviations of this so-called Boussinesq approximation do affect the characteristic nature of pattern formation upon the appearance of convection [5,41-43]. However, the intensity of the nonequilibrium fluctuations in convection-free states below the convective instability appears to be much less sensitive to deviations from the Boussinesq approximation. Segrè et al. have analyzed and measured the temperature fluctuations in liquid toluene subjected to temperature gradients up to $220 \mathrm{~K} \mathrm{~cm}^{-1}$ [12]. At the higher value of the temperature gradient some of the thermophysical properties varied significantly over the height of the fluid layer. Nevertheless, the intensity of the observed nonequilibrium temperature fluctuations was equal to the intensity predicted when all thermophysical-property values are taken at their average value in the fluid layer. Similar results were obtained by Ahlers, measuring the critical Rayleigh number for the onset of RayleighBénard convection and the heat transfer slightly above the threshold [42].

We note that in the derivation of the Boussinesq equations it is also assumed that the adiabatic temperature gradient $\left(\alpha \bar{T}_{0} / c_{P}\right) g$, where $c_{P}$ is the isobaric specific heat capacity and $\bar{T}_{0}$ the average temperature in the fluid layer, is small and can be neglected compared to the magnitude of the imposed temperature gradient. In practice, this is a very good approximation $[38,39]$. In addition, in the Boussinesq Eq. (4b) the coefficient multiplying $\nabla^{2} \theta$ is usually identified with the thermal diffusivity of the fluid $D_{T}$. For consistency, we have also expressed the prefactor of the second random noise term in Eq. (5b) in terms of the same diffusivity $D_{T}[39]$. 


\section{Bulk structure factor of a fluid subjected to a stationary tem- perature gradient}

Starting from the fluctuating Boussinesq equations, we first derive an expression for the structure factor of a fluid subjected to a stationary temperature gradient in the presence of gravity but in the absence of any boundary conditions. Althought the resulting expression for such a bulk structure factor will only be meaningful for negative Rayleigh numbers, it will enable us to develop a procedure most suited for subsequent incorporation of boundary effects.

In the absence of any boundary conditions, a temporal and spatial Fourier transformation can be applied to Eqs. (4) to obtain a set of equations for the fluctuations in the vertical component of the velocity $w(\omega, \mathbf{q})$ and for the fluctuations in the temperature $\theta(\omega, \mathbf{q})$ as a function of the frequency $\omega$ and the wavevector $\mathbf{q}$ :

$$
\left(\begin{array}{cc}
-q^{2}\left(\mathrm{i} \omega+\nu q^{2}\right) & \alpha g q_{\|}^{2} \\
\nabla T_{0} & \mathrm{i} \omega+D_{T} q^{2}
\end{array}\right)\left(\begin{array}{c}
w(\omega, \mathbf{q}) \\
\theta(\omega, \mathbf{q})
\end{array}\right)=\left(\begin{array}{c}
F_{1}(\omega, \mathbf{q}) \\
F_{2}(\omega, \mathbf{q})
\end{array}\right),
$$

where $q_{\|}=\sqrt{q_{x}^{2}+q_{y}^{2}}$ represents the magnitude of the component of the wavevector $\mathbf{q}$ in the XY-plane, i.e., the component of $\mathbf{q}$ perpendicular to the temperature gradient. We note that in this paper the parallel and perpendicular directions are defined with respect to the horizontal plane, as done by most researchers in the field $[8,26,27,31]$, but unlike the notation used by Segrè et al. [20,21]. The Fourier transforms $F_{1}(\omega, \mathbf{q})$ and $F_{2}(\omega, \mathbf{q})$ of the random noise terms can be expressed as a function of the Fourier transforms of the random stress tensor and the random heat flow:

$$
\begin{gathered}
F_{1}(\omega, \mathbf{q})=\frac{\mathrm{i}}{\rho}\left\{q^{2}[\mathbf{q} \cdot \delta \mathbf{T}(\omega, \mathbf{q})]_{z}-q_{z} \mathbf{q} \cdot[\mathbf{q} \cdot \delta \mathbf{T}(\omega, \mathbf{q})]\right\}, \\
F_{2}(\omega, \mathbf{q})=-\mathrm{i} \frac{D_{T}}{\lambda} \mathbf{q} \cdot \delta \mathbf{Q}(\omega, \mathbf{q}) .
\end{gathered}
$$

The solution for $w(\omega, \mathbf{q})$ and $\theta(\omega, \mathbf{q})$ can be readily obtained by inverting the matrix in Eq. (6), so that:

$$
\left(\begin{array}{l}
w(\omega, \mathbf{q}) \\
\theta(\omega, \mathbf{q})
\end{array}\right)=\frac{\left(\begin{array}{cc}
\mathrm{i} \omega+D_{T} q^{2} & -\alpha g q_{\|}^{2} \\
-\nabla T_{0} & -q^{2}\left(\mathrm{i} \omega+\nu q^{2}\right)
\end{array}\right)}{q^{2}\left[\omega-\mathrm{i} \Omega_{+}(\mathbf{q})\right]\left[\omega-\mathrm{i} \Omega_{-}(\mathbf{q})\right]}\left(\begin{array}{l}
F_{1}(\omega, \mathbf{q}) \\
F_{2}(\omega, \mathbf{q})
\end{array}\right),
$$


where we have introduced the quantities:

$$
\Omega_{ \pm}(\mathbf{q})=\frac{1}{2} q^{2}\left[\left(\nu+D_{T}\right) \pm \sqrt{\left(\nu-D_{T}\right)^{2}-4 g \alpha \nabla T_{0} \frac{q_{\|}^{2}}{q^{6}}}\right] .
$$

Stricly speaking, in the expression (9) for the decay rates $\Omega_{ \pm}(\mathbf{q})$ the temperature gradient $\nabla T_{0}$ should be identified with the effective temperature gradient $\nabla T_{0}+\left(\alpha \bar{T}_{0} / c_{P}\right) g$, as shown by Segrè et al. [20]. However, as mentioned in Section 2 , the contribution $\left(\alpha \bar{T}_{0} / c_{P}\right) g$ from the adiabatic temperature gradient is neglected in the Boussinesq approximation. In the limit $g \rightarrow 0$, we have $\Omega_{+}=\nu q^{2}$ and $\Omega_{-}=D_{T} q^{2}$, which are the decay rates associated with the transverse-velocity fluctuations $w$ and the temperature fluctuations $\theta$, respectively.

In the Boussinesq approximation, density fluctuations are caused only by temperature fluctuations, while pressure fluctuations are neglected. Consequently, the structure factor $S(\omega, \mathbf{q})$ will be related to the autocorrelation function of the temperature fluctuations:

$$
\left\langle\theta^{*}(\omega, \mathbf{q}) \theta\left(\omega^{\prime}, \mathbf{q}^{\prime}\right)\right\rangle=\frac{1}{\alpha^{2} \rho^{2}} S(\omega, \mathbf{q})(2 \pi)^{4} \delta\left(\omega-\omega^{\prime}\right) \delta\left(\mathbf{q}-\mathbf{q}^{\prime}\right) .
$$

To deduce the autocorrelation function $\left\langle\theta^{*}(\omega, \mathbf{q}) \theta\left(\omega^{\prime}, \mathbf{q}^{\prime}\right)\right\rangle$ of the temperature fluctuations from Eqs. (8), we need the correlation functions for the Langevin noise terms $F_{1}(\omega, \mathbf{q})$ and $F_{2}(\omega, \mathbf{q})$, defined by Eqs. (5). In nonequilibrium fluctuating hydrodynamics it is assumed that the random stress tensor and the random heat flow retain their local-equilibrium values $[8,44,45]$. Thus, as in equilibrium, we shall consider both $\delta \mathrm{T}$ as $\delta \mathbf{Q}$ as "white" noise. Their autocorrelation functions are short ranged in time and in space, being proportional to delta functions. Fourier transforming the explicit expressions for the correlation functions of $\delta \mathbf{T}(\mathbf{r}, t)$ and $\delta \mathbf{Q}(\mathbf{r}, t)$ as, for instance, given by Eqs. (3.12) in Ref. [8], and using the definition $(5)$ of $F_{1}(\omega, \mathbf{q})$ and $F_{2}(\omega, \mathbf{q})$, we obtain:

$$
\begin{aligned}
& \left\langle F_{1}^{*}(\omega, \mathbf{q}) F_{1}\left(\omega^{\prime}, \mathbf{q}^{\prime}\right)\right\rangle=2 k_{\mathrm{B}} T \frac{\nu}{\rho} q_{\|}^{2} q^{4}(2 \pi)^{4} \delta\left(\omega-\omega^{\prime}\right) \delta\left(\mathbf{q}-\mathbf{q}^{\prime}\right), \\
& \left\langle F_{2}^{*}(\omega, \mathbf{q}) F_{2}\left(\omega^{\prime}, \mathbf{q}^{\prime}\right)\right\rangle=\frac{2 k_{\mathrm{B}} T^{2} \lambda}{\rho^{2} c_{P}^{2}} q^{2}(2 \pi)^{4} \delta\left(\omega-\omega^{\prime}\right) \delta\left(\mathbf{q}-\mathbf{q}^{\prime}\right), \\
& \left\langle F_{1}^{*}(\omega, \mathbf{q}) F_{2}\left(\omega^{\prime}, \mathbf{q}^{\prime}\right)\right\rangle=\left\langle F_{2}^{*}(\omega, \mathbf{q}) F_{1}\left(\omega^{\prime}, \mathbf{q}^{\prime}\right)\right\rangle=0,
\end{aligned}
$$

where $k_{\mathrm{B}}$ represents Boltzmann's constant. Combining Eqs. (8), (10) and (11) we obtain for the dynamic structure factor $S(\omega, \mathbf{q})$ of a nonequilibrium fluid 
in the Boussinesq approximation the following expression:

$$
S(\omega, \mathbf{q})=S_{\mathrm{E}}\left\{\frac{2 D_{T} q^{2}\left(\omega^{2}+\nu^{2} q^{4}\right)}{\left[\omega^{2}+\Omega_{+}^{2}(\mathbf{q})\right]\left[\omega^{2}+\Omega_{-}^{2}(\mathbf{q})\right]}+\frac{2 \nu\left(c_{P} / T\right) \nabla T_{0}^{2} q_{\|}^{2}}{\left[\omega^{2}+\Omega_{+}^{2}(\mathbf{q})\right]\left[\omega^{2}+\Omega_{-}^{2}(\mathbf{q})\right]}\right\},
$$

where $S_{\mathrm{E}}$ represents the intensity of the fluctuations in thermodynamic equilibrium:

$$
S_{\mathrm{E}}=\rho^{2} \kappa_{T} k_{\mathrm{B}} T \frac{\gamma-1}{\gamma}
$$

Here $\gamma$ denotes the heat-capacity ratio $c_{P} / c_{V}$ and $\kappa_{T}$ is the isothermal compressibility. In deriving Eq. (12) we have employed the thermodynamic relation $\alpha^{2} D_{T}=[(\gamma-1) / \gamma] \lambda \kappa_{T} / T$.

The equation for the Rayleigh spectrum of a fluid subjected to a stationary temperature gradient including the contribution from gravity was first derived by Segrè et al. [20]. We recover the same result, but without the (negligible) contribution from the adiabatic temperature gradient. In the limit $g \rightarrow 0$, Eq. (12) reduces to the expression of the Rayleigh spectrum of a fluid subjected to a stationary temperature gradient, first obtained by Kirkpatrick et al. [6], and subsequently reproduced by other investigators [7-9]. The nonequilibrium structure factor, as given by Eq. (12), is anisotropic, and it depends on the magnitude of the horizontal component $q_{\|}$of the scattering vector directly and through the decay rates $\Omega_{ \pm}(\mathbf{q})$.

In this paper we shall focus our attention on the static structure factor:

$$
S(\mathbf{q})=(2 \pi)^{-1} \int d \omega S(\omega, \mathbf{q})
$$

which determines the total intensity of Rayleigh scattering [46]. For subsequent use, we find it convenient to introduce dimensionless wave numbers $\tilde{q}=q L$ and $\tilde{q}_{\|}=q_{\|} L$, where $L$ is the finite height of the fluid layer. Upon substituting Eq. (9) for $\Omega_{ \pm}(\mathbf{q})$ into Eq. (12) and integrating the resulting expression for $S(\omega, \mathbf{q})$, we obtain:

$$
S(\mathbf{q})=S_{\mathrm{E}}\left\{1+\tilde{S}_{\mathrm{NE}}^{0} \frac{\tilde{q}_{\|}^{2}}{\left(\tilde{q}^{6}-R \tilde{q}_{\|}^{2}\right)}\right\}
$$

In Eq. (15) $\tilde{S}_{\mathrm{NE}}^{0}$ represents the strength of the nonequilibrium enhancement of the structure factor, which is given by:

$$
\tilde{S}_{\mathrm{NE}}^{0}=\frac{\sigma R}{\sigma+1}+\frac{(\sigma-1)\left(c_{P} / T\right) L^{4}}{\nu^{2}-D_{T}^{2}}\left(\nabla T_{0}\right)^{2}
$$


where $\sigma=\nu / D_{T}$ is the Prandtl number. Since we have not yet considered any finite-size effects, Eqs. (15) and (16) do not depend explicitly on the height L. Equations (15) and (16) are identical to Eq. (2.35) in Ref. [20]. The term in Eq. (16) proportional to $R$ is related to the adiabatic temperature gradient and is in practice negligibly small. Hence, the intensity of nonequilibrium fluctuations continues to be proportional to $\left(\nabla T_{0}\right)^{2}$ in the presence of gravity.

Note that (15) can only be valid for negative Rayleigh number $R$, i.e., when the fluid layer is heated from above so that $\nabla T_{0}>0$. For any $R>0$, the nonequilibrium contribution to the structure factor will always diverge at some finite value of the horizontal component $q_{\|}$of the scattering vector. The appearance of this divergence is a direct consequence of us having performed the calculation without taking into account boundary conditions. As will be demonstrated in the next section, when boundary conditions are taken into account, a different expression for $S(\mathbf{q})$ is obtained. This new expression will be valid not only for negative Rayleigh numbers, but also for a finite interval of positive Rayleigh numbers, up to some critical Rayleigh number $R_{\mathrm{c}}$.

\section{Modification of the nonequilibrium structure factor due to finite- size effects}

Since in practice the fluid layer is confined between two horizontal plates separated by a (small) distance $L$, the nonequilibrium structure factor will be affected by the presence of boundary conditions in the Z-direction. In some previous publications, we have evaluated finite-size effects on the nonequilibrium structure factor neglecting gravity [24,25,47]. A major conclusion of this previous work is that finite-size effects appear at wave numbers where in practice the nonequilibrium structure factor will also be affected by gravity. Hence, for the interpretation of experimental measurements it becomes imperative to incorporate both finite-size effects and gravity effects simultaneously. This task is implemented in the present section.

As in our previous publication [24], we again apply a Fourier transformation of the fluctuating Boussinesq equations (4) in space and in time, but restricting the spatial Fourier transformation to the XY-plane. We thus obtain the following set of linear stochastic differential equations:

$\left(\begin{array}{rr}\mathrm{i} \omega\left[\frac{d^{2}}{d z^{2}}-q_{\|}^{2}\right]-\nu\left[\frac{d^{2}}{d z^{2}}-q_{\|}^{2}\right]^{2} & \alpha g q_{\|}^{2} \\ \nabla T_{0} & \mathrm{i} \omega-D_{T}\left[\frac{d^{2}}{d z^{2}}-q_{\|}^{2}\right]\end{array}\right)\left(\begin{array}{l}w\left(\omega, \mathbf{q}_{\|}, z\right) \\ \theta\left(\omega, \mathbf{q}_{\|}, z\right)\end{array}\right)=\left(\begin{array}{l}F_{1}\left(\omega, \mathbf{q}_{\|}, z\right) \\ F_{2}\left(\omega, \mathbf{q}_{\|}, z\right)\end{array}\right)$, 
where $\mathbf{q}_{\|}$is the wavevector in the XY-plane. The random noise terms $F_{1}\left(\omega, \mathbf{q}_{\|}, z\right)$ and $F_{2}\left(\omega, \mathbf{q}_{\|}, z\right)$ in Eqs. (17) are related to the partial Fourier transforms $\delta \mathrm{T}\left(\omega, \mathbf{q}_{\|}, z\right)$ of the random stress tensor and $\delta \mathbf{Q}\left(\omega, \mathbf{q}_{\|}, z\right)$ of the random heat flux. The actual expressions are a bit complicated and can be found elsewhere [25].

As is often done in the literature [26,35], for the sake of simplicity, we adopt stress-free boundary conditions for the vertical velocity and perfectly conducting walls for the temperature, so that:

$$
\begin{aligned}
& \theta\left(\omega, \mathbf{q}_{\|}, z\right)=0 \quad \text { at } \quad z=0, L, \\
& w\left(\omega, \mathbf{q}_{\|}, z\right)=0 \quad \text { at } \quad z=0, L, \\
& \frac{d^{2}}{d z^{2}} w\left(\omega, \mathbf{q}_{\|}, z\right)=0 \quad \text { at } \quad z=0, L \text {. }
\end{aligned}
$$

Note that these boundary conditions imply the absence of any possible fluctuations in the temperature and velocity of the fluid adjacent to the walls. As is well known, stress-free boundary conditions represent a fluid bounded by two free surfaces, which is a rather unrealistic case $[35,48]$. For the realistic case of a fluid confined between two rigid surfaces, no-slip boundary conditions in the velocity are more appropriate. For the particular case of $g=0$, we have evaluated the nonequilibrium structure factor using both stress-free [24] and no-slip [25] boundary conditions. While there are numerical differences between the two solutions, the dependence of the nonequilibrium structure factor on the wave number $q$ appears to be qualitatively the same for the two sets of boundary conditions. For mathematical simplicity, we evaluate here the nonequilibrium structure factor using stress-free boundary conditions in the expectation that also in the presence of gravity the more realistic no-slip boundary conditions will not modify the qualitative nature of the wave-number dependence of the structure factor.

To search for a solution of Eq. (17), we represent $w\left(\omega, \mathbf{q}_{\|}, z\right)$ and $\theta\left(\omega, \mathbf{q}_{\|}, z\right)$ as a series expansion in a complete set of eigenfunctions of the differential operator in Eq. (17), satisfying the boundary conditions (18) [24]. Because of the simplicity of the boundary conditions considered here, an appropriate set of eigenfunctions is the Fourier sine basis in the $[0, L]$ interval [35]. We thus assume:

$$
\left(\begin{array}{c}
w\left(\omega, \mathbf{q}_{\|}, z\right) \\
\theta\left(\omega, \mathbf{q}_{\|}, z\right)
\end{array}\right)=\sum_{N=1}^{\infty}\left(\begin{array}{c}
A_{N}\left(\omega, \mathbf{q}_{\|}\right) \\
B_{N}\left(\omega, \mathbf{q}_{\|}\right)
\end{array}\right) \sin \left(\frac{N \pi z}{L}\right)
$$

To deduce the coefficients $A_{N}\left(\omega, \mathbf{q}_{\|}\right)$and $B_{N}\left(\omega, \mathbf{q}_{\|}\right)$from Eq. (17), we also have to represent the random noise terms $F_{1}\left(\omega, \mathbf{q}_{\|}, z\right)$ and $F_{2}\left(\omega, \mathbf{q}_{\|}, z\right)$ as a 
Fourier sine series:

$$
\left(\begin{array}{c}
F_{1}\left(\omega, \mathbf{q}_{\|}, z\right) \\
F_{2}\left(\omega, \mathbf{q}_{\|}, z\right)
\end{array}\right)=\sum_{N=1}^{\infty}\left(\begin{array}{c}
F_{1, N}\left(\omega, \mathbf{q}_{\|}\right) \\
F_{2, N}\left(\omega, \mathbf{q}_{\|}\right)
\end{array}\right) \sin \left(\frac{N \pi z}{L}\right)
$$

where we have introduced the set of random functions $F_{1, N}\left(\omega, \mathbf{q}_{\|}\right)$and $F_{2, N}\left(\omega, \mathbf{q}_{\|}\right)$, which are the projections of the random noise terms over the eigenfunction basis. They are given by:

$$
\left(\begin{array}{c}
F_{1, N}\left(\omega, \mathbf{q}_{\|}\right) \\
F_{2, N}\left(\omega, \mathbf{q}_{\|}\right)
\end{array}\right)=\frac{2}{L} \int_{0}^{L}\left(\begin{array}{c}
F_{1}\left(\omega, \mathbf{q}_{\|}, z\right) \\
F_{2}\left(\omega, \mathbf{q}_{\|}, z\right)
\end{array}\right) \sin \left(\frac{N \pi z}{L}\right) d z
$$

Representing the random noise terms by Eq. (20), one readily deduces from Eq. (17) expressions for the coefficients of the Fourier series $A_{N}\left(\omega, \mathbf{q}_{\|}\right)$and $B_{N}\left(\omega, \mathbf{q}_{\|}\right)$. To calculate the structure factor in the Boussinesq approximation, we only need the coefficients $B_{N}\left(\omega, \mathbf{q}_{\|}\right)$for the temperature fluctuations. Substituting Eqs. (19) and (20) into Eq. (17) we obtain:

$B_{N}\left(\omega, \mathbf{q}_{\|}\right)=\frac{-\nabla T_{0} F_{1, N}\left(\omega, \mathbf{q}_{\|}\right)-\left(\frac{N^{2} \pi^{2}}{L^{2}}+q_{\|}^{2}\right)\left[\mathrm{i} \omega+\nu\left(\frac{N^{2} \pi^{2}}{L^{2}}+q_{\|}^{2}\right)\right] F_{2, N}\left(\omega, \mathbf{q}_{\|}\right)}{\left(\frac{N^{2} \pi^{2}}{L^{2}}+q_{\|}^{2}\right)\left(\omega-\mathrm{i} \Omega_{+}\left(N, q_{\|}\right)\right)\left(\omega-\mathrm{i} \Omega_{-}\left(N, q_{\|}\right)\right)}$,

where, similarly to Eq. (9), we have introduced decay rates $\Omega_{ \pm}\left(N, q_{\|}\right)$, which are now given by:

$$
\Omega_{ \pm}\left(N, q_{\|}\right)=\frac{1}{2}\left(\frac{N^{2} \pi^{2}}{L^{2}}+q_{\|}^{2}\right)\left[\left(\nu+D_{T}\right) \pm \sqrt{\left(\nu-D_{T}\right)^{2}-\frac{4 g \alpha \nabla T_{0} q_{\|}^{2}}{\left(\frac{N^{2} \pi^{2}}{L^{2}}+q_{\|}^{2}\right)^{3}}}\right]
$$

Since the differential operator in Eq. (17) depends only on the modulus of $\mathbf{q}_{\|}$, the problem has cylindrical symmetry and the solution will only depend on the magnitude $q_{\|}$of the two-dimensional wavevector $\mathbf{q}_{\|}$.

In analogy to Eq. (10), the relationship between the dynamic structure factor $S\left(\omega, q_{\|}, z, z^{\prime}\right)$ and the autocorrelation function of the temperature fluctuations is now given by:

$$
\left\langle\theta^{*}\left(\omega, \mathbf{q}_{\|}, z\right) \theta\left(\omega^{\prime}, \mathbf{q}_{\|}^{\prime}, z^{\prime}\right)\right\rangle=\frac{(2 \pi)^{3}}{\alpha^{2} \rho^{2}} S\left(\omega, q_{\|}, z, z^{\prime}\right) \delta\left(\omega-\omega^{\prime}\right) \delta\left(\mathbf{q}_{\|}-\mathbf{q}_{\|}^{\prime}\right)
$$

To obtain the structure factor $S\left(\omega, q_{\|}, z, z^{\prime}\right)$ we need the correlation functions between the different projections of the random noise terms. These have been calculated in a previous publication with the result [24]: 


$$
\begin{aligned}
\left\langle F_{1, N}^{*}\left(\omega, \mathbf{q}_{\|}\right) \cdot F_{1, M}\left(\omega^{\prime}, \mathbf{q}_{\|}^{\prime}\right)\right\rangle & =2 k_{\mathrm{B}} T \frac{\nu}{\rho} \frac{2}{L} q_{\|}^{2}\left(q_{\|}^{2}+\frac{N^{2} \pi^{2}}{L^{2}}\right)^{2} \delta_{N M} \\
& \times(2 \pi)^{3} \delta\left(\omega-\omega^{\prime}\right) \delta\left(\mathbf{q}_{\|}-\mathbf{q}_{\|}^{\prime}\right), \\
\left\langle F_{1, N}^{*}\left(\omega, \mathbf{q}_{\|}\right) \cdot F_{2, M}\left(\omega^{\prime}, \mathbf{q}_{\|}^{\prime}\right)\right\rangle & =\left\langle F_{2, N}^{*}\left(\omega, \mathbf{q}_{\|}\right) \cdot F_{1, M}\left(\omega^{\prime}, \mathbf{q}_{\|}^{\prime}\right)\right\rangle=0, \\
\left\langle F_{2, N}^{*}\left(\omega, \mathbf{q}_{\|}\right) \cdot F_{2, M}\left(\omega^{\prime}, \mathbf{q}_{\|}^{\prime}\right)\right\rangle & =\frac{2 k_{\mathrm{B}} T^{2} \lambda}{\rho^{2} c_{P}^{2}} \frac{2}{L}\left(q_{\|}^{2}+\frac{N^{2} \pi^{2}}{L^{2}}\right) \delta_{N M} \\
& \times(2 \pi)^{3} \delta\left(\omega-\omega^{\prime}\right) \delta\left(\mathbf{q}_{\|}-\mathbf{q}_{\|}^{\prime}\right) .
\end{aligned}
$$

As in the case of the absence of any boundary conditions, we continue to assume that the correlation functions between the different components of the random current tensor and the random heat flux retain their equilibrium values. This assumption remains valid as long as $L$ is a macroscopic distance, much larger than the molecular distances in the fluid.

In this paper we are interested in the static structure factor $S\left(q_{\|}, z, z^{\prime}\right)=$ $(2 \pi)^{-1} \int d \omega S\left(\omega, q_{\|}, z, z^{\prime}\right)$. With the information presented above, this quantity can be readily calculated. In analogy to Eq. (15) for $S(\mathbf{q})$ in the absence of any boundary conditions, the modified nonequilibrium structure factor can be written in the form:

$$
S\left(q_{\|}, z, z^{\prime}\right)=S_{\mathrm{E}}\left[\delta\left(z-z^{\prime}\right)+\tilde{S}_{\mathrm{NE}}^{0} \tilde{S}_{\mathrm{NE}}\left(q_{\|}, z, z^{\prime}\right)\right]
$$

where $S_{\mathrm{E}}$ and $\tilde{S}_{\mathrm{NE}}^{0}$ are again given by Eqs. (13) and (16), respectively, while $\tilde{S}_{\mathrm{NE}}\left(q_{\|}, z, z^{\prime}\right)$ incorporates the finite-size effects and is given by:

$$
\tilde{S}_{\mathrm{NE}}\left(q_{\|}, z, z^{\prime}\right)=\frac{2 \tilde{q}_{\|}^{2}}{L} \sum_{N=1}^{\infty} \frac{\sin \left(\frac{N \pi z}{L}\right) \sin \left(\frac{N \pi z^{\prime}}{L}\right)}{\left(\tilde{q}_{\|}^{2}+N^{2} \pi^{2}\right)^{3}-R \tilde{q}_{\|}^{2}} .
$$

The first term in Eq. (26) is again the static structure factor of a fluid in thermodynamic equilibrium, which is not affected by any finite-size effects [46]. The second term in Eq. (26) represents the nonequilibrium enhancement of the structure factor. This nonequilibrium enhancement is proportional to $\left(\nabla T_{0}\right)^{2}$ through the expression (16) for $\tilde{S}_{\mathrm{NE}}^{0}$, it depends on the gravitational acceleration constant $g$ through the appearance of the Rayleigh number in Eqs. (16) and (26), and it depends on the finite height $L$ of the fluid layer explicitly in Eq. (26) and also through $\tilde{q}_{\|}=q_{\|} L$. In the limit $R \rightarrow 0$ we recover the expression for $S\left(q_{\|}, z, z^{\prime}\right)$ presented in a previous publication [24].

It is important to note that Eq. (27) is valid for both negative and positive Rayleigh numbers, provided that

$$
R<R_{\mathrm{c}}=\frac{27 \pi^{4}}{4}
$$


For $R>R_{\mathrm{c}}$ there always exist values of $q_{\|}$for which right-hand side of Eq. (27) diverges. Of course, the value $R_{\mathrm{c}}=27 \pi^{4} / 4$ equals the well-known value obtained form a linear stability analysis of the Boussinesq equations with noslip boundary conditions [35]. As expected, for $R \geq R_{\mathrm{c}}$ the present calculation breaks down because the fluctuations around the conductive state are no longer small.

Equation (27) for the normalized nonequilibrium enhancement $\tilde{S}_{\mathrm{NE}}\left(\tilde{q}_{\|}, z, z^{\prime}\right)$ represents our principal result for the combined effects of gravity and finitesize on the nonequilibrium structure factor of the fluid. The remaining part of this paper is concerned with an analysis of some of the physical consequences that follow from Eq. (27).

The double Fourier series contained in Eq. (27), when it is convergent, can be easily summed. To do so, we first find the roots of the denominator for $N^{2}$. We then split the inverse of the product of three binomials as a sum of three terms, and we finally obtain for $\tilde{S}_{\mathrm{NE}}\left(\tilde{q}_{\|}, z, z^{\prime}\right)$ :

$$
\tilde{S}_{\mathrm{NE}}\left(\tilde{q}_{\|}, z, z^{\prime}\right)=\frac{2}{3 L} \tilde{q}_{\|}^{2 / 3} \sum_{N=1}^{\infty} \sum_{i=0}^{2} \frac{\sin \left(\frac{N \pi z}{L}\right) \sin \left(\frac{N \pi z^{\prime}}{L}\right)}{\lambda_{i}^{2}\left[N^{2} \pi^{2}-\left(\frac{\lambda_{i}}{\tilde{q}_{\|}^{4 / 3}}-1\right) \tilde{q}_{\|}^{2}\right]},
$$

where $\lambda_{i}$ for $i=0,1,2$ are the three complex cubic roots of $R$ :

$$
\lambda=|R|^{1 / 3}\left(\begin{array}{c}
\exp \text { i } \frac{\psi}{3} \\
\exp \text { i } \frac{\psi+2 \pi}{3} \\
\exp \text { i } \frac{\psi+4 \pi}{3}
\end{array}\right)
$$

In Eq. (30) $\psi$ represents the phase of $R$ when considered as a complex number: $\psi=0$ for $R>0$ and $\psi=\pi$ for $R<0$. This procedure is only valid for $R \neq 0$. However, the finite-size effects on the structure factor for the particular case $R=0$ have already been considered in a previous publication [24], where the sum of the series in Eq. (27) for this particular case has been performed by a different method. Hence, we do not need to re-evaluate $\tilde{S}_{\mathrm{NE}}$ for the case $R=0$. To implement the summation in Eq. (29) we consider the relation:

$$
\begin{aligned}
& \sum_{N=1}^{\infty} \frac{1}{N^{2}-\mu} \sin \left(\frac{N \pi z}{L}\right) \sin \left(\frac{N \pi z^{\prime}}{L}\right)= \\
& \frac{-\pi}{4 \sqrt{\mu}} \frac{\cos \left(\frac{\pi \sqrt{\mu}}{L}\left(L-\left|z-z^{\prime}\right|\right)\right)-\cos \left(\frac{\pi \sqrt{\mu}}{L}\left(L-\left|z+z^{\prime}\right|\right)\right)}{\sin (\pi \sqrt{\mu})} .
\end{aligned}
$$

This equation can be obtained from formula 1.445 in Ref. [49] and it is valid 
for $z, z^{\prime} \in[0, L]$ and for any complex number $\mu$, provided that in the righthand side of (31) the square root of $\mu$ with positive real part is chosen. Now, the sum of the double Fourier series contained in Eq. (27) can be performed, but the result is a long expression, not particularly informative, which can be easily obtained by the reader. Explicit expressions to be obtained upon further integration of $\tilde{S}_{\mathrm{NE}}\left(\tilde{q}, z, z^{\prime}\right)$ will be presented in the following section.

\section{Consequences for light scattering and shadowgraph experiments}

\subsection{Nonequilibrium structure factor probed in experiments}

The nonequilibrium fluctuations can be obserbed in small-angle light-scattering experiments. For a discussion of such light-scattering experiments we consider an experimental arrangement like the ones employed at the University of Maryland [9-12] and by Vailati and Giglio at the University of Milan [22,23]. A schematic representation of such a light-scattering experiment is shown in Fig. 1. The scattering medium is a thin horizontal fluid layer bounded by two parallel plates whose temperatures can be controlled independently so as to establish a temperature gradient across the fluid layer. The temperature gradient can be parallel or antiparallel to the direction of gravity. The horizontal plates are furnished with windows allowing laser light to propagate through the fluid in the direction parallel to the gravity and to the temperature gradient. Light scattered over an angle $\phi$ arises from fluctuations with a wave number such that [46]:

$$
q=2 q_{0} \sin (\phi / 2)
$$

where $q_{0}$ is the wave number of the incident light inside the scattering medium. To observe any nonequilibrium fluctuations one needs to observe the scattered light at small wave numbers and, hence, at small scattering angles.

From electromagnetic theory [46] it follows that the scattering intensity $S(\mathbf{q})$ is obtained from an integration of the structure factor over the scattering volume, so that [24,27]:

$$
S\left(q_{\|}, q_{\perp}\right)=\frac{1}{L} \int_{0}^{L} \int_{0}^{L} \mathrm{e}^{-i q_{\perp}\left(z-z^{\prime}\right)} S\left(q_{\|}, z, z^{\prime}\right) d z d z^{\prime}
$$

In Eq. (33) we have assumed that the scattering volume extends over the full height of the fluid layer as is the case in small-angle light scattering from a thin fluid layer [12]. In this situation scattered light received in the collecting pinhole of the detector indeed arises from all points illuminated by the laser 


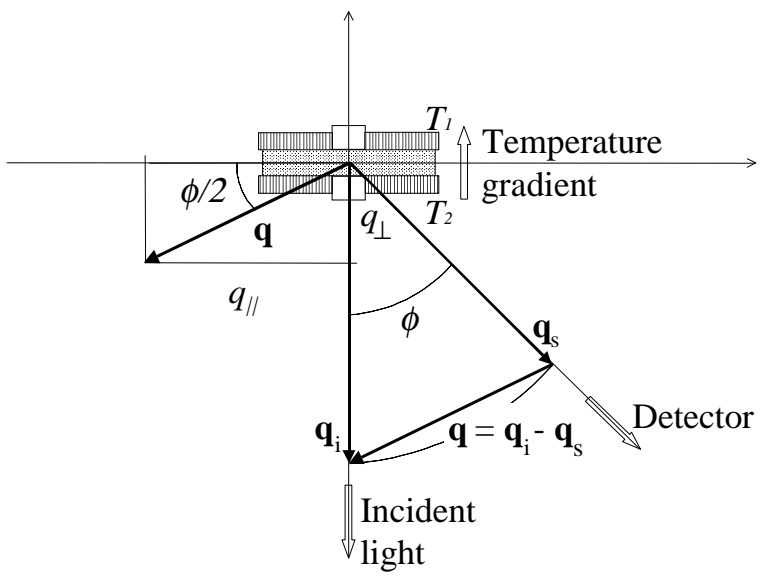

Fig. 1. Schematic representation of a nonequilibrim light-scattering experiment. $\mathbf{q}_{\mathbf{i}}$ is the wave vector of the incident light and $\mathbf{q}_{\mathrm{s}}$ is the wave vector of the scattered light. The magnitude $q=\left|\mathbf{q}_{\mathrm{i}}-\mathbf{q}_{\mathrm{s}}\right|$ of the scattering wave vector is related to the scattering angle $\phi$ by $q=2 q_{0} \sin (\phi / 2)$, where $q_{0}$ is the magnitude of the wave vector $\mathbf{q}_{i}$ of the incident light inside the fluid.

beam inside the fluid layer. From Eq. (32) and the geometrical arrangement shown in Fig. 1, we note that $q_{\|}$and $q_{\perp}$ in an actual light-scattering experiment are not independent variables, because they are related to the scattering angle, $\phi$, by:

$$
\begin{aligned}
& q_{\|}=q \cos (\phi / 2)=2 q_{0} \sin (\phi / 2) \cos (\phi / 2), \\
& q_{\perp}=q \sin (\phi / 2)=2 q_{0} \sin ^{2}(\phi / 2) .
\end{aligned}
$$

Equation (34) shows that for actual small-angle light-scattering experiments one may use the approximation $q_{\|} \simeq q, q_{\perp} \simeq 0$.

An alternative promising experimental technique for measuring the intensity, though not the temporal spectrum, of nonequilibrium fluctuations is quantitative shadowgraph analysis [28,50-53]. The experimental arrangement is similar to the one depicted in Fig. 1 but, instead of a laser beam, an extended uniform monochromatic light source is employed to illuminate the sample. Then many shadowgraph images of a plane perpendicular to the temperature gradient are obtained with a CCD detector, which measures the spatial distribution of intensity $I(\mathbf{x})$, where $\mathbf{x}$ is a two-dimensional position vector in the imaging plane. For each image a shadowgraph signal is defined by:

$$
i(\mathbf{x})=\frac{I(\mathbf{x})-I_{0}(\mathbf{x})}{I_{0}(\mathbf{x})}
$$

where $I_{0}(\mathbf{x})$ is the blank intensity distribution, when there are no fluctuations in the index of refraction of the sample. In practice $I_{0}(\mathbf{x})$ is calculated by averaging over many shadowgraph images, so that fluctuations cancel out and 
the resulting $I_{0}(\mathbf{x})$ contains only contributions from nonuniform illumination of the sample. From physical and geometrical optics one can demonstrate that the modulus squared of the two-dimensional Fourier transform of the shadowgraph signal, $|i(\mathbf{q})|^{2}$, after taking an azimuthal average, can be expressed as $[50,51,53]$ :

$$
\overline{|i(\mathbf{q})|^{2}}(q)=\hat{T}(q) S\left(q_{\|}=q, q_{\perp}=0\right)
$$

where the overline indicates the azimuthal average, in which case the result depends only on the modulus $q_{\|}$of the two-dimensional Fourier vector $\mathbf{q}$. The factor $\hat{T}(q)$ represents an optical transfer function, which can be derived from the optical arrangement used to produce the shadowgraph pictures; it includes contributions from the response of the CCD detector and the density dependence of the refractive index $[50,51,53]$. Therefore, applying a two-dimensional Fourier transform to the shadowgraph images, one can deduce the structure factor of the fluid as a function of the wave number $q$ at $q_{\perp}=0$.

There exist an equivalence between small-angle light scattering and shadowgraphy, in the sense that both methods give us $S\left(q_{\|}=q, q_{\perp}=0\right)$. For light scattering, $q$ is the scattering wavevector as given by (32), whereas for shadowgraph techniques $q$ is the modulus of the two-dimensional Fourier vector in the imaging plane. As mentioned by Bodenschatz et al., what it is measured in the experiments is a kind of vertical average of the fluctuations [51].

To obtain an explicit expression for the structure factor $S\left(q_{\|}, q_{\perp}\right)$, we substitute Eq. (26) with $\tilde{S}_{\mathrm{NE}}\left(q_{\|}, z, z^{\prime}\right)$ given by Eq. (27) into Eq. (33), implement the summation by using the relation (31) and perform the double integration in Eq. (33). Introducing again dimensionless wave numbers $\tilde{q}_{\|}=q_{\|} L$ and $\tilde{q}_{\perp}=q_{\perp} L$, we thus obtain:

$$
S\left(\tilde{q}_{\|}, \tilde{q}_{\perp}\right)=S_{\mathrm{E}}\left\{1+\tilde{S}_{\mathrm{NE}}^{0} \tilde{S}_{\mathrm{NE}}\left(\tilde{q}_{\|}, \tilde{q}_{\perp}\right)\right\}
$$

with

$$
\begin{aligned}
\tilde{S}_{\mathrm{NE}}\left(\tilde{q}_{\|}, \tilde{q}_{\perp}\right) & =\frac{\tilde{q}_{\|}^{2}}{\tilde{q}^{6}-R \tilde{q}_{\|}^{2}} \\
& +\frac{2 \tilde{q}_{\|}}{3 R} \sum_{j=0}^{2} \frac{\lambda_{j} \sqrt{\lambda_{j}-\tilde{q}_{\|}^{4 / 3}}}{\left[\tilde{q}^{2}-\lambda_{j} \tilde{q}_{\|}^{2 / 3}\right]^{2}} \frac{\cos \left(\tilde{q}_{\perp}\right)-\cos \left(\tilde{q}_{\|}^{1 / 3} \sqrt{\lambda_{j}-\tilde{q}_{\|}^{4 / 3}}\right)}{\sin \left(\tilde{q}_{\|}^{1 / 3} \sqrt{\lambda_{j}-\tilde{q}_{\|}^{4 / 3}}\right)} .
\end{aligned}
$$

In Eq. (37), $S_{\mathrm{E}}$ and $S_{\mathrm{NE}}^{0}$ are again given by Eqs. (13) and (16), respectively, while $\lambda_{i}$ in Eq. (38) again represent the three complex cubic roots of $R$ defined by Eq. (30). In thermodynamic equilibrium $\nabla T_{0}=0$ and $R=0$, so that $S\left(\tilde{q}_{\|}, \tilde{q}_{\perp}\right)=S_{\mathrm{E}}$ and we recover from Eq. (37) the well-known isotropic equilibrium structure factor given by Eq. (13). 
If we retain only the first term on the right-hand side of Eq. (38) and substitute it in Eq. (37) we recover the expression (15) for the bulk structure factor of the fluid. As noted in Section 3, this expression for the bulk structure factor breaks down for any positive value of the Rayleigh number because of a divergence at $q^{6}=q_{\|}^{2} R L^{-4}$. The second term in Eq. (38) for $\tilde{S}_{\mathrm{NE}}\left(\tilde{q}_{\|}, \tilde{q}_{\perp}\right)$, containing a sum over the three complex cubic roots of $R$, represents the finitesize effects on the nonequilibrium structure factor and depends on the height $L$ of the fluid layer. Although perhaps not immediately transparent, it can be verified that this second term when multiplied by $\tilde{S}_{\mathrm{NE}}^{0}$ vanishes in the limit $L \rightarrow \infty$. Moreover, for any finite value of $L$, the divergence of the first term in Eq. (38) at $q^{6}=q_{\|}^{2} R L^{-4}$ for positive $R$ is now exactly compensated by a similar divergence in the second term of Eq. (38) for the same wave number, since $\lambda_{0}=R^{1 / 3}$ for positive $R$. Thus $\tilde{S}_{\mathrm{NE}}\left(\tilde{q}_{\|}, \tilde{q}_{\perp}\right)$, as given by Eq. (38), is continuous at $q^{6}=q_{\|}^{2} R L^{-4}$ for positive $R$. However, due to the presence of a sine term in the denominator, new divergences appear when:

$$
\tilde{q}_{\|}^{1 / 3} \sqrt{R^{1 / 3}-\tilde{q}_{\|}^{4 / 3}}=N \pi
$$

for $N=0,1, \ldots$ For $N=0$ this new divergence is again compensated due to the presence of the term $\sqrt{R^{1 / 3}-\tilde{q}_{\|}^{4 / 3}}$ in the numerator. But for $N=1$, there is no compensation and the contribution (38) to the nonequilibrium enhancement of the structure factor diverges. Since condition (39) for $N=1$ is satisfied when $R=\left(\tilde{q}_{\|}^{2}+\pi^{2}\right)^{3} / \tilde{q}_{\|}^{2}$, this divergence will appear for any Rayleigh number larger than the critical Rayleigh number $R_{\mathrm{c}}=27 \pi^{4} / 4$. For $R=R_{\mathrm{c}}$, condition (39) is satisfied at a critical wave number $\tilde{q}_{\| \mathrm{c}}=\pi / \sqrt{2}$. We thus recover from an analysis of the divergences in the nonequilibrium structure factor the well-known results of the linear stability theory for a fluid layer bounded by two free surfaces [35]. A more detailed analysis of the behavior of our solution for the nonequilibrium structure factor for positive $R$ near $R_{\mathrm{c}}$ will be presented in Section 6 .

As mentioned earlier, for the interpretation of low-angle scattering or shadowgraph experiments one may consider the approximation $q_{\|} \approx q, q_{\perp} \approx 0$. In the limit $q_{\perp} \rightarrow 0$, Eq. (38) for $\tilde{S}_{\mathrm{NE}}\left(\tilde{q}_{\|}, \tilde{q}_{\perp}\right)$ can be slightly simplified; in this approximation the nonequilibrium enhancement $\tilde{S}_{\mathrm{NE}}(\tilde{q})$ will depend only on $\tilde{q}$, which is the experimentally relevant quantity. In the remainder of this paper the small-angle approximation will always be assumed. We note that all the divergent features discussed above remain the same in this approximation.

It is interesting to look at the asymptotic behavior of the nonequilibrium enhancement for $q \rightarrow 0$ and for $q \rightarrow \infty$. From Eq. (38) it can be readily shown that:

$$
\tilde{S}_{\mathrm{NE}}(\tilde{q}) \stackrel{\tilde{q} \rightarrow 0}{\longrightarrow} \frac{17}{20160} \tilde{q}^{2}
$$


and

$$
\tilde{S}_{\mathrm{NE}}(\tilde{q}) \stackrel{\tilde{q} \rightarrow \infty}{\longrightarrow} \frac{1}{\tilde{q}^{4}}
$$

We note from Eqs. (40) and (41) that the limiting behavior of $\tilde{S}_{\mathrm{NE}}(\tilde{q})$ for $q \rightarrow 0$ and $q \rightarrow \infty$ does not depend on the value of the Rayleigh number $R$.

\subsection{Interpretation of experimental results}

In accordance with Eq. (37), the product $\tilde{S}_{\mathrm{NE}}^{0} \tilde{S}_{\mathrm{NE}}(\tilde{q})$ represents the nonequilibrium enhancement of the structure factor with $\tilde{S}_{\mathrm{NE}}^{0}$ given by Eq. (16) and $\tilde{S}_{\mathrm{NE}}(\tilde{q})$ given by Eq. (38) with $\tilde{q}_{\perp} \rightarrow 0$. From Eq. (16) we see that the prefactor $\tilde{S}_{\mathrm{NE}}^{0}$ depends on various thermophysical properties of the fluid and on the magnitude of the applied temperature gradient, but $\tilde{S}_{\mathrm{NE}}^{0}$ is independent of the wave number $\tilde{q}$. Hence, to evaluate the wave-number dependence of the nonequilibrium enhancement of the structure factor, we only need to focus our attention on $\tilde{S}_{\mathrm{NE}}(\tilde{q})$.

Assuming $\tilde{q}_{\perp}=0$, we have evaluated $\tilde{S}_{\mathrm{NE}}(\tilde{q})$ from Eq. (38). In Fig. 2 we show on a double-logarithmic scale $\tilde{S}_{\mathrm{NE}}(\tilde{q})$ as a function of $\tilde{q}$ for three different values of the Rayleigh number, namely for a large negative value, $R=-5000$, for a value $R \simeq 0$ which corresponds to the case investigated in a previous publication [24] and for a value $R=600$ which is close to the critical value $R_{\mathrm{c}} \simeq 656$. At larger values of $\tilde{q}, \tilde{S}_{\mathrm{NE}}(\tilde{q})$ varies as $\tilde{q}^{-4}$, independent of the Rayleigh number, in agreement with Eq. (41). Upon decrease of $\tilde{q}, \tilde{S}_{\mathrm{NE}}(\tilde{q})$ goes through a maximum and for very small values of $\tilde{q}, \tilde{S}_{\mathrm{NE}}(\tilde{q})$ decreases as $\tilde{q}^{2}$, in agreement with Eq. (40). For positive $R, \tilde{S}_{\mathrm{NE}}(\tilde{q})$ develops a prominent peak close to $\tilde{q}_{\mathrm{c}}=\pi / \sqrt{2}$, which diverges as $R \rightarrow R_{\mathrm{c}}$, as further discussed in Section 6.1. Hence, a major effect of the additive noise terms in the fluctuating Boussinesq equations is the appearance of (fluctuating) patterns in the fluid, even below the convective instability as discussed by some other investigators $[54,55]$.

Sengers and coworkers have measured the nonequilibrium fluctuations in liquid toluene [10-12] and in liquid $n$-hexane [56]. These experiments correspond to Rayleigh numbers from $-25,000$ to $-300,000$ at dimensionless wave numbers ranging from $\tilde{q}=640$ down to $\tilde{q}=345$. The experiments have provided an accurate confirmation of the $q^{-4}$ dependence of the intensity of nonequilibrium fluctuations in this range of wave numbers.

Giglio and coworkers have measured the intensity of nonequilibrium fluctuations for negative Rayleigh numbers down to wave numbers $\tilde{q}$ of order unity, both with ultra-low angle light-scattering experiments $[22,23]$ and from quanti- 


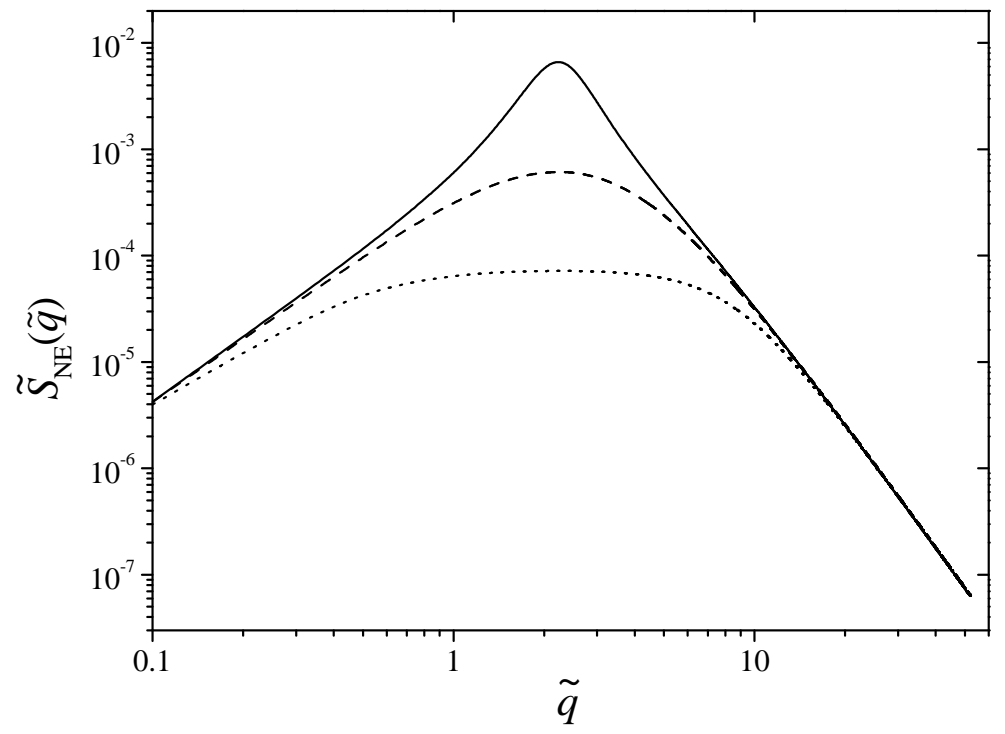

Fig. 2. Normalized nonequilibrium enhancement $\tilde{S}_{\mathrm{NE}}(\tilde{q})$ of the structure factor as given by Eq. (38), as a function of the dimensionless wave number $\tilde{q}$ for three different values of the Rayleigh number $R$. The solid curve is for $R=600$, which is near the convective instability, the dashed curve is for $R \approx 0$ and the dotted curve for $R=-5000$.

tative analysis of shadowgraph images $[52,53]$. They actually measured the intensity of nonequilibrium concentration fluctuations in a liquid mixture. However, due to the similar structure of the underlying hydrodynamic equations, the $q$ dependence of the contribution of nonequilibrium concentration fluctuations to the structure factor in a liquid mixture is expected to be similar to the $q$ dependence of the contribution of nonequilibrium temperature fluctuations to the structure factor of a one-component fluid [13,47]. Giglio and coworkers have not only confirmed the $q^{-4}$ dependence of the nonequilibrium structure factor, but they have also observed the crossover to a region of $\tilde{q}$ close to unity where the nonequilibrium structure factor is independent of $\tilde{q}$, in agreement with the flat region indicated in Fig. 2 for $\tilde{S}_{\mathrm{NE}}(\tilde{q})$ at large negative Rayleigh numbers.

$\mathrm{Wu}$ et al. have measured the nonequilibrium structure factor in a layer of fluid carbon dioxide, at a pressure of about $3 \mathrm{MPa}$, near the convective instability. For positive Rayleigh numbers, $\tilde{S}_{\mathrm{NE}}(\tilde{q})$ strongly depends on the parameter $\epsilon=\left(R-R_{\mathrm{c}}\right) / R_{\mathrm{c}}$ which measures the distance from the Rayleigh-Bénard instability. In Fig. 3 we show $\tilde{S}_{\mathrm{NE}}(\tilde{q})$ as a function of $\tilde{q}$ close to $\tilde{q}_{\mathrm{c}}=\pi / \sqrt{2}$ as calculated from Eq. (38) for $\epsilon=-0.03, \epsilon=-0.01$ and $\epsilon=-0.005$. We did not attempt to make a quantitative comparison with the measurements of $\mathrm{Wu}$ et al., because the slip-free boundary conditions adopted in this paper do not correspond to the experimental boundary conditions. However, a comparison of Fig. 3 with Fig. 3 in Ref. [28] shows that our solution yields 


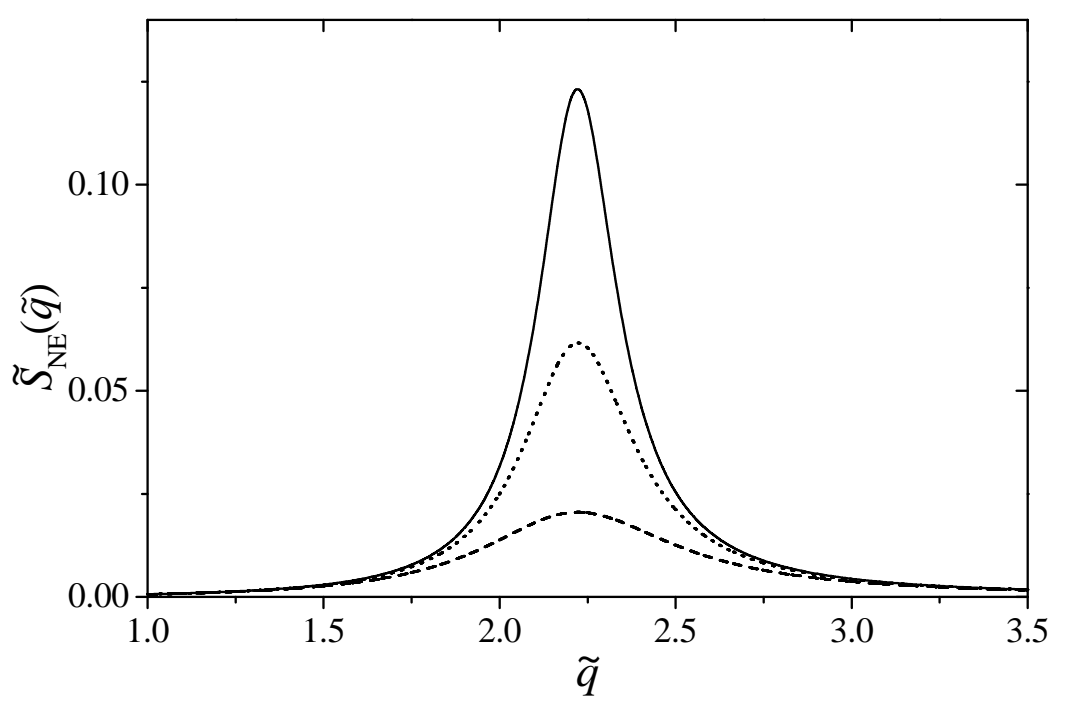

Fig. 3. Normalized nonequilibrium enhancement $\tilde{S}_{\mathrm{NE}}(\tilde{q})$ of the structure factor as given by Eq. (38), as a function of the dimensionless wave number $\tilde{q}$ for three different values of the distance $\epsilon$ to the convective instability. The solid curve is for $\epsilon=-5 \times 10^{-3}$, the dotted curve is for $\epsilon=-10^{-2}$ and the dashed curve for $\epsilon=-3 \times 10^{-2}$.

a good qualitative representation of the $q$ dependence of the nonequilibrium structure factor as measured by Wu et al. We conclude that our solution of the linearized fluctuating Boussinesq equations for the nonequilibrium structure factor is consistent with the characteristic features of the nonequilibrium structure factor observed in experiments for both negative and positive Rayleigh numbers.

\section{Nonequilibrium fluctuations close to the convective instability}

The nature of thermal noise near the convective instability has been the subject of studies by many investigators $[1,4,26,27,31,36]$. Hence, it is of interest to make a comparison of those results with our solution for the intensity of temperature fluctuations for thermal nonequilibrium states. It turns out that for an analysis of $\tilde{S}_{\mathrm{NE}}(\tilde{q})$ near the convective instability it is more convenient not to perform the summation of the series in Eq. (27). Instead, substituting Eq. (27) into Eq. (33) and integrating the terms in the series individually, we can write $\tilde{S}_{\mathrm{NE}}(\tilde{q})$ as:

$$
\tilde{S}_{\mathrm{NE}}(\tilde{q})=4 \sum_{N=1}^{\infty} \frac{\tilde{q}^{2}}{\left(\tilde{q}^{2}+N^{2} \pi^{2}\right)^{3}-R \tilde{q}^{2}} \frac{1-\cos (N \pi)}{N^{2} \pi^{2}}
$$

which is equivalent to the expression (38). 


\subsection{Divergence of nonequilibrium fluctuations at the convective instability}

Zaitsev and Shliomis [1] were the first to compute thermal fluctuations in a fluid layer subjected to a stationary temperature gradient near the convective instability. Using linear perturbation theory they found that the structure factor diverges as $\left(R_{\mathrm{c}}-R\right)^{-1}$. The same divergence follows from our solution for $\tilde{S}_{\mathrm{NE}}(\tilde{q})$. To reproduce the divergence we first calculate the value $\tilde{q}_{\max }$ corresponding to the maximum of $\tilde{S}_{\mathrm{NE}}(\tilde{q})$ by expanding $\tilde{q}_{\max }$ in powers of $\epsilon=\left(R-R_{\mathrm{c}}\right) / R_{\mathrm{c}}$ around $\pi / \sqrt{2}$. Differentiating the expression (42) for $\tilde{S}_{\mathrm{NE}}(\tilde{q})$ and demanding the derivative to vanish, we find that the position of the maximum corresponds to:

$$
\begin{aligned}
\tilde{q}_{\max } & =\frac{\pi}{\sqrt{2}}+\epsilon^{2} \frac{81 \sqrt{2} \pi}{8} \sum_{N=2}^{\infty} \frac{\left(N^{2}-1\right)\left(1+2 N^{2}\right)^{2}(1-\cos N \pi)}{N^{2}\left[\left(1+2 N^{2}\right)^{3}-27\right]^{2}}+O\left(\epsilon^{3}\right) \\
& \approx \frac{\pi}{\sqrt{2}}+6.324 \times 10^{-4} \epsilon^{2}
\end{aligned}
$$

Substituting Eq. (43) into Eq. (42) we conclude that the structure factor, which is proportional to the intensity of the scattered light, diverges when the convective instability is approached such that:

$$
\tilde{S}_{\mathrm{NE}}\left(\tilde{q}_{\max }\right)=\frac{-54 \pi^{2}}{\epsilon}+\frac{16}{\pi^{6}} \sum_{N=2}^{\infty} \frac{1-\cos N \pi}{N^{2}\left[\left(1+2 N^{2}\right)^{3}-27\right]}+O(\epsilon) .
$$

We thus recover the linear divergence of $\tilde{S}_{\mathrm{NE}}$ as a function of $\left(R-R_{\mathrm{c}}\right)^{-1}$ obtained by Zaitsev and Shilomis [1] and confirmed by Swift and Hohenberg $[4,36]$. Linear fluctuation theory amounts to a mean-field theory of fluctuations. Extremely close to the instability nonlinear effects will cause a smearing out of the sharp mean-field transition $[2,4,36]$, but this effect will only be noticeable for very small values of $|\epsilon| \lesssim 2.9 \times 10^{-5}$ [40]. Hence, observation of this linear divergence is possible in experiments [28]. Deviations from linear fluctuation theory have been observed by Scherer et al. in the case of electroconvection [57].

\subsection{The most unstable mode and the Swift-Hohenberg approximation}

The approximation scheme used by Zaitsev and Shliomis and by Swift and Hohenberg is equivalent to retaining only the term $N=1$ in the series expansion (42) for $\tilde{S}_{\mathrm{NE}}(\tilde{q})$. Note that, for $N=1$, when $R$ is close to $R_{\mathrm{c}}$ and $q_{\|}$is close to $q_{\mathrm{c}}$, the denominator in the corresponding term in Eq. (27) approaches

zero. Therefore, in this situation the term for $N=1$ is much larger than the 
terms for any other value of $N$. Consequently, when $R \lesssim R_{\mathrm{c}}$ and $q \simeq q_{\mathrm{c}}$, truncating the series (42) at $N=1$ yields a very good approximation. We thus deduce from our solution for the structure factor as measured in low-angle light scattering or shadowgraph experiments:

$$
S(\tilde{q})=\frac{8 S_{\mathrm{E}} \tilde{S}_{\mathrm{NE}}^{0}}{\pi^{2}} \frac{1}{\frac{\left(\tilde{q}^{2}+\pi^{2}\right)^{3}}{\tilde{q}^{2}}-R},
$$

where the equilibrium contribution to $S(\tilde{q})$ has been neglected ( cf., Eq. (37)). This is usually called the most-unstable-mode approximation in the literature [1,4]. The denominator in Eq. (45) is zero at $R=R_{\mathrm{c}}=27 \pi^{4} / 4$ and $\tilde{q}_{\|}=\tilde{q}_{\| \mathrm{c}}=\pi / \sqrt{2}$. Expanding the denominator in powers of $\tilde{q}_{\|}^{2}$ around $\tilde{q}_{\| \mathrm{c}}^{2}$, one obtains:

$$
S(\tilde{q})=\frac{4 S_{\mathrm{E}} \tilde{S}_{\mathrm{NE}}^{0}}{\tilde{q}_{\mathrm{c}}^{2} R_{\mathrm{c}}} \frac{1}{\tilde{\xi}_{0}^{4}\left(\tilde{q}^{2}-\tilde{q}_{\mathrm{c}}^{2}\right)^{2}-\epsilon},
$$

where, following the notation of Hohenberg and Swift [36], we have introduced the parameter $\tilde{\xi}_{0}^{2}=2 /\left(\sqrt{3} \pi^{2}\right)$. Equation (46) is proportional to the correlation function of the Swift-Hohenberg ( $\mathrm{SH}$ ) model below the convective instability, when nonlinear terms are negligible $[4,36]$. The results of $\mathrm{SH}$ refer to the autocorrelation of a field $\psi\left(\mathbf{r}_{\|}, t\right)$, which is a generalized two-dimensional order parameter introduced by them to describe the convective instability as a nonequilibrium phase transition. If we compare the term $N=1$ in Eq. (27) with Eq. (2.10) in Ref. [36], we identify our $S_{\mathrm{NE}}(q)$ as proportional to the equal-time autocorrelation function of the field $\psi$. Therefore the $\mathrm{SH}$ model can be obtained as an approximation close to the convective instability from our exact results for $S\left(q_{\|}=q, q_{\perp}=0\right)$, as given by Eqs. (37) and (38). As discussed by SH, Eq. (46) is valid for both "stress-free" and "no-slip" boundary conditions, but the numerical values of the constants $R_{\mathrm{c}}, q_{\| \mathrm{c}}$ and $\tilde{\xi}_{0}^{2}$ do depend on the boundary conditions (see Table I in Ref. [36]). An interesting feature of the SH model is that is a "Lagrangian" model, because the autocorrelation function (46) can be obtained from a simple probability functional for the field $\psi\left(\mathbf{r}_{\|}\right)$, as is discussed in detail in the relevant literature $[4,36]$.

To compare our more complete solution of the fluctuating Boussinesq equations for $\tilde{S}_{\mathrm{NE}}(\tilde{q})$ with the various approximations proposed for the structure factor close to the convective instability, we have plotted in Fig. $4 \tilde{S}_{\mathrm{NE}}(\tilde{q})$ as a function of $\tilde{q}$. The solid line represents $\tilde{S}_{\mathrm{NE}}(\tilde{q})$ as given by our exact solution (38), the dotted curve (hardly visible) the most-unstable-mode approximation (45) and the dashed curve the SH-model approximation (46). All three curves correspond to a fluid layer subjected to a temperature gradient such that $\epsilon=-0.01$. From Fig. 4 it is evident that Eq. (45) yields a very good approximation although at large $\tilde{q}$ deviations appear. The reason is that 


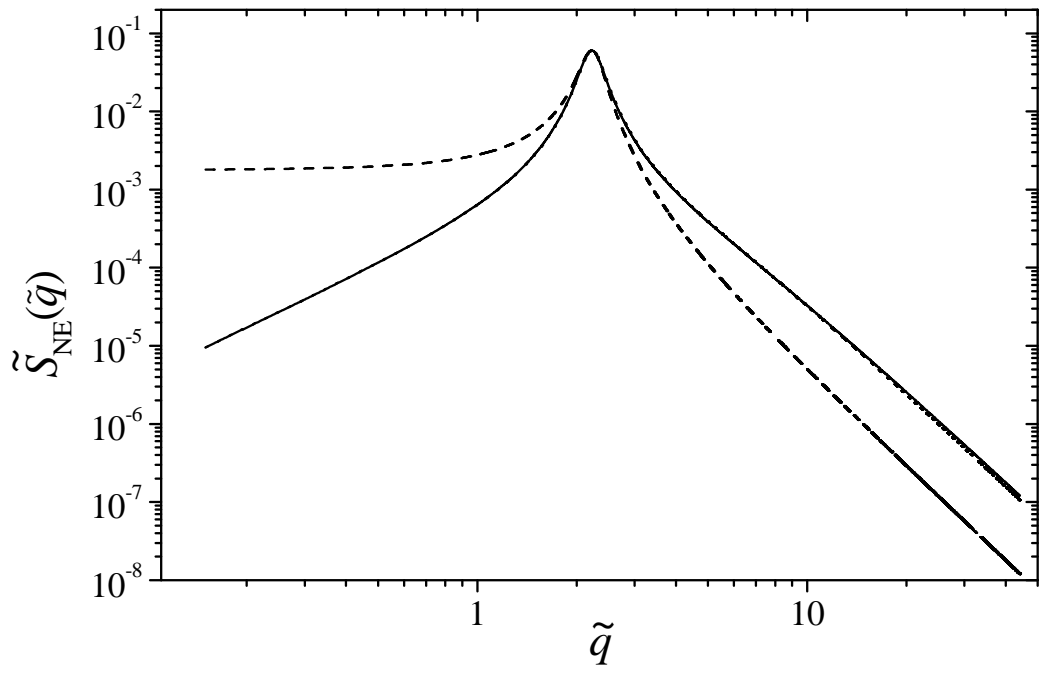

Fig. 4. Normalized nonequilibrium enhancement $\tilde{S}_{\mathrm{NE}}(\tilde{q})$ of the structure factor as a function of the dimensionless wave number $\tilde{q}$ near the convective instability, $\epsilon=-10^{-2}$. The solid line represents the exact expression as given by Eq. (38). The dotted curve (indistingishable from the former, except asymptotically for large q) represents the most-unstable-mode approximation given by Eq. (45). The dashed curve represents the Swift-Hohenberg approximation given by Eq. (46).

$\tilde{S}_{\mathrm{NE}}(\tilde{q})$ for large $\tilde{q}$ should vary as $\tilde{q}^{-4}$, while Eq. (45) yields $\left(8 / \pi^{2}\right) \tilde{q}^{-4}$ for large $\tilde{q}$.

Many authors have represented the $q$ dependence of the structure factor near the instability in terms of a Lorentzian profile centered at $\tilde{q}_{\mathrm{c}}$ with a width proportional to $\epsilon[26,27,31,36]$, and $\mathrm{Wu}$ et al. have also analyzed their experimental data in terms of a Lorentzian profile [28]. However, our actual solution (38) does not yield a Lorentzian profile, although it can be obtained by expanding the denominator in Eq. (45) not in powers of $\tilde{q}^{2}$ around $\tilde{q}_{\mathrm{c}}^{2}$, but in powers of $\tilde{q}$ around $\tilde{q}_{\mathrm{c}}$. The problem is that a Lorentzian does not lead to the proper asymptotic behavior for either small $q$ or large $q$. It even leads to an apparent divergence when one tries to calculate the power of the fluctuations by integrating $S(q)$ over all two-dimensional wave vectors $q[28,36]$. We conclude that it would be much better to represent the experimental data in terms of an equation like Eq. (45), which is not a Lorentzian.

\subsection{Power of thermal fluctuations}

Another feature of nonequilibrium fluctuations close to the convective instability that has been studied theoretically and experimentally is the so-called power of the thermal fluctuations, $\left\langle\delta T^{2}\right\rangle$. The mean-square amplitude $\left\langle\delta T^{2}\right\rangle$ of the temperature fluctuations is related to the integral of the structure factor 
measured in the experiments:

$$
\left\langle\delta T^{2}\right\rangle=\frac{1}{\alpha^{2} \rho^{2}} \frac{1}{L} \int S(q) \frac{d^{2} \mathbf{q}}{(2 \pi)^{2}},
$$

which corresponds to a vertical average of the real-space temperature autocorrelation function. Due to the symmetry of the problem this quantity does not depend on the point $\mathbf{x}_{\|}$in the horizontal plane at which is evaluated. In Eq. (47) the prefactor $1 /\left(\alpha^{2} \rho^{2}\right)$ accounts for the conversion from density fluctuations to temperature fluctuations (cf., Eqs. (10) and (26)) and the factor $1 / L$ results from the vertical average (compare Eq. (33) with Eq. (A14) in Ref. [36]). From Eq. (42) we note that in terms of our solution of the fluctuating Boussinesq equations (neglecting the equilibrium contribution):

$$
\left\langle\delta T^{2}\right\rangle=\frac{S_{\mathrm{E}} \tilde{S}_{\mathrm{NE}}^{0}}{\alpha^{2} \rho^{2} L \pi^{2}} \sum_{N=1}^{\infty} \int_{0}^{\infty} \frac{\tilde{q}^{2} 2 \pi q d q}{\left(\tilde{q}^{2}+N^{2} \pi^{2}\right)^{3}-R \tilde{q}^{2}} \frac{1-\cos (N \pi)}{N^{2} \pi^{2}} .
$$

The integral in (48) can be performed analytically, but the result is long and not particularly interesting. Asymptotically close to the convective instability we obtain:

$$
\left\langle\delta T^{2}\right\rangle \simeq \frac{S_{\mathrm{E}} \tilde{S}_{\mathrm{NE}}^{0}}{\alpha^{2} \rho^{2} L^{3}} \frac{\sqrt{3}}{R_{\mathrm{c}} \sqrt{-\epsilon}} .
$$

Thus the mean-square amplitude $\left\langle\delta T^{2}\right\rangle$ calculated from our solution will diverge as $1 / \sqrt{-\epsilon}$, which is the kind of divergence observed experimentally by Ahlers and coworkers [28,51]. In Eq. (49), $\tilde{S}_{\mathrm{NE}}^{0}$ has to be evaluated at the critical temperature gradient. It is interesting to note that the $\mathrm{SH}$ approximation gives for $\left\langle\delta T^{2}\right\rangle$ the same asymptotic behavior, Eq. (49), than the exact solution.

\section{Correlations in real space}

In this section we study the real-space behavior of the autocorrelation function of the nonequilibrium density fluctuations. We also shall make contact with the third approach dealing with nonequilibrium fluctuations that was mentioned in the Introduction. To calculate the autocorrelation function in real space we apply an inverse Fourier transform in the horizontal plane to the structure factor as given by Eq. (26). The equilibrium contribution gives a triple delta function. The nonequilibrium contribution gives a nontrivial contribution, which we denote as $G_{\mathrm{NE}}\left(r_{\|}, z, z^{\prime}\right)$ and which is equal to:

$$
G_{\mathrm{NE}}\left(r_{\|}, z, z^{\prime}\right)=\frac{S_{\mathrm{E}} \tilde{S}_{\mathrm{NE}}^{0}}{2 \pi} \int_{0}^{\infty} q_{\|} J_{0}\left(q_{\|} r_{\|}\right) \tilde{S}_{\mathrm{NE}}\left(q_{\|}, z, z^{\prime}\right) d q_{\|}
$$




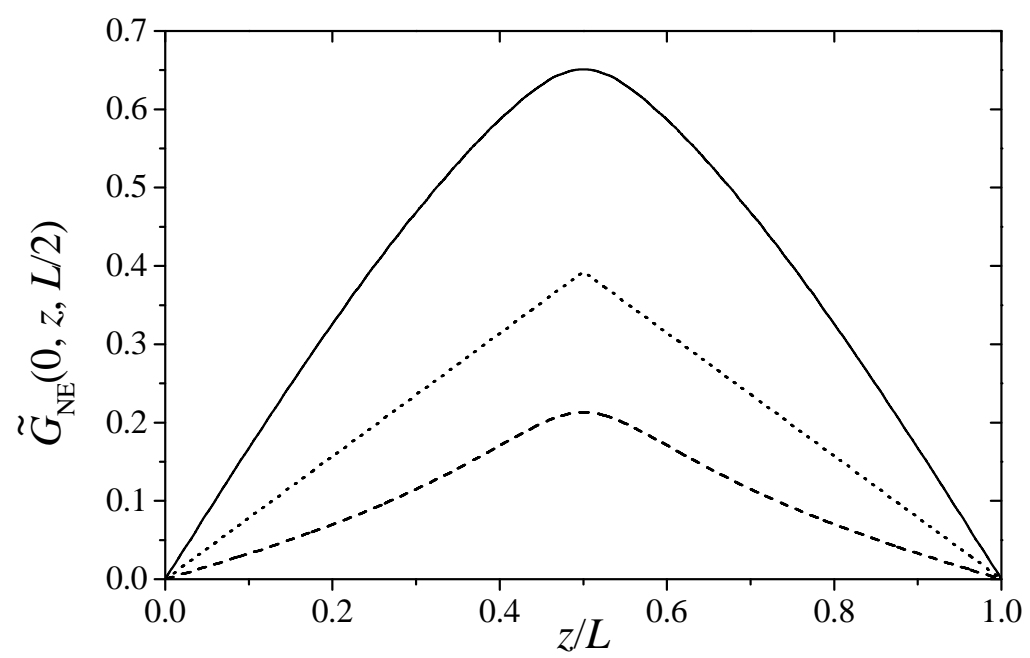

Fig. 5. Normalized dimensionless nonequilibrium correlation function $\tilde{G}_{\mathrm{NE}}\left(r_{\|}, z, z^{\prime}\right)$ in the Z-direction $\left(r_{\|}=0\right)$ as a function of $z / L$ for $z^{\prime}=L / 2$. The solid curve is for $R=500$, the dotted lines are for $R=0$ and the dashed curve is for $R=-2000$.

where $J_{0}(x)$ is the Bessel function of the first kind and order zero. Note that due to the cylindrical symmetry of the problem the correlation in real space depends only on the cylindrical radial coordinate $\left(r_{\|}\right)$. It is difficult to study the integral (50) in the general case, but there are a couple of particular cases for which integral (50) can be evaluated explicitly or where simple approximations can be made so that the real-space behavior of the correlation function can be studied in detail.

\subsection{Correlations in the vertical direction}

The first interesting case for which the long-range part of the real-space correlation function $G_{\mathrm{NE}}\left(r_{\|}, z, z^{\prime}\right)$ can be studied is for $r_{\|}=0$, i.e., along the vertical Z-direction. In this particular case, the correlation function $G_{\mathrm{NE}}$ will only depend on the variables $z$ and $z^{\prime}$ and on the Rayleigh number $R$. Close to the convective instability, the behavior of the real-space correlation function will be dominated by the most unstable mode. From Eq. (27) we note that $G_{\mathrm{NE}}$ will be proportional to the product $\sin (\pi z / L) \sin \left(\pi z^{\prime} / L\right)$. Therefore, the concavity of $G_{\mathrm{NE}}\left(0, z, z^{\prime}\right)$ as a function of $z$ for fixed value of $z^{\prime}$ is negative. For the interesting case $R=0, G_{\mathrm{NE}}\left(0, z, z^{\prime}\right)$ was evaluated exactly in a previous publication [24]. It turns out that the concavity of $G_{\mathrm{NE}}\left(0, z, z^{\prime}\right)$ changes at $R=0$, being negative for positive $R$ and positive for negative $R$ (except for a small region near $z=z^{\prime}$ ). In Fig. 5 we have plotted the dimensionless nonequilibrium correlation function, $\tilde{G}_{\mathrm{NE}}=G_{\mathrm{NE}} L^{3} /\left(S_{\mathrm{E}} \tilde{S}_{\mathrm{NE}}^{0}\right)$, as a function of 
$z$, for $z^{\prime}=L / 2, r_{\|}=0$ and for three values of the Rayleigh number $R$. The dotted lines correspond to $R=0$, the solid curve corresponds to a positive $R$ and the dashed curve to a negative $R$. The change of the concavity of the function at $R=0$ is evident. This feature can be investigated analytically since, for small values of the Rayleigh number, $G_{\mathrm{NE}}\left(0, z, z^{\prime}\right)$ can be readily expanded in powers of $R$ yielding:

$$
\begin{aligned}
G_{\mathrm{NE}}\left(0, z, z^{\prime}\right) \simeq \frac{S_{\mathrm{E}} \tilde{S}_{\mathrm{NE}}^{0}}{2 \pi L^{3}} & \left\{\frac{\left|z+z^{\prime}\right|-\left|z-z^{\prime}\right|}{8 L}-\frac{z z^{\prime}}{4 L^{2}}\right. \\
& \left.+\frac{R}{1350}\left[B_{6}\left(\frac{\left|z-z^{\prime}\right|}{2 L}\right)-B_{6}\left(\frac{\left|z+z^{\prime}\right|}{2 L}\right)\right]\right\}
\end{aligned}
$$

where $B_{6}(x)$ is the Bernoulli polynomial of the 6th degree (actually, it is possible to obtain a systematic expansion of $G_{\mathrm{NE}}\left(0, z, z^{\prime}\right)$ in terms of the Bernoulli polynomials, but the resulting series is not very informative). For $R=0$, we recover from Eq. (51) the expression obtained for the spatial dependence of the correlation function in a previous publication (see Eq. (25) and Fig 2 in Ref. [24]). The change of concavity of $G_{\mathrm{NE}}\left(0, z, z^{\prime}\right)$ at $R=0$ is also evident from Eq. (51). We note that for $R=0$ the function $G_{\mathrm{NE}}\left(0, z, z^{\prime}\right)$ has a kink at $z=z^{\prime}$, indicating the presence of a discontinuity in the derivative at this point. For any other value of $R<R_{\mathrm{c}}$ the function and its derivative are continuous.

From Fig 5 we also note that the nonequilibrium real-space equal-time correlation function is always positive, has a maximum at $z=z^{\prime}$ and decreases monotonically from this maximum reaching zero at both ends of the $[0, L]$ interval. Thus, we conclude that the real-space correlation function in the vertical direction is nonlocal, long ranged and does not involve any intrinsic length scale, i.e., the correlation encompasses the entire system only to be cut off by the finite size of the system itself. Hence, the presence of the temperature gradient induces long-range correlations in the direction coincident with the temperature gradient, as has been elucidated by previous investigators, mainly on the basis of the nonequilibrium heat-diffusion equation $[14,58,59]$. Considering only the heat-diffusion equation means that one neglects any coupling between temperature and velocity fluctuations. This approach neglects also any gravity effects and is restricted to fluctuations with $q_{\|}=0$. The existence of long-range correlations in the direction of the temperature gradient has been verified by numerical integration [17], by computer simulation [18], by lattice-gas-automaton dynamics [19] and by a master-equation approach [60]. Our result for the correlation function along the direction of the temperature gradient is in agreement with that obtained by Malek Mansour and coworkers [14,17], as is also evident form a comparison of our Fig. 5 for $R=0$ with Fig. 1 in Ref. [17]. Some of these authors have also considered a coupling between temperature and velocity fluctuations [61]. But to simplify the hydrodynamic equations they made the assumption that the thermal expansion coefficient of the fluid vanishes. This is equivalent to assum- 


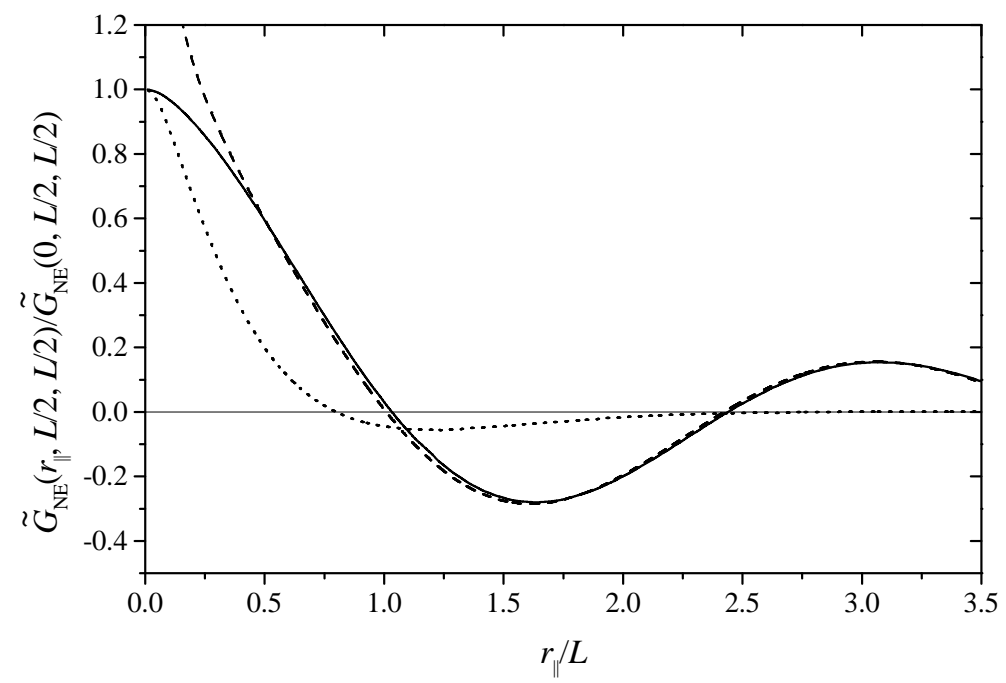

Fig. 6. Normalized dimensionless nonequilibrium correlation function $\tilde{G}_{\mathrm{NE}}\left(r_{\|}, z, z^{\prime}\right)$ in the central plane of the liquid layer $\left(z=z^{\prime}=L / 2\right)$, near the convective instability, as a function of $r_{\|} / L$, divided by its value at $r_{\|}=0$. The solid curve is for $\epsilon=-10^{-2}$; the dashed curve is the asymptotic approximation, Eq. (53), for the same value of $\epsilon$. The dotted curve is for $\epsilon=-0.7$.

ing that density fluctuations are caused only by pressure fluctuations. Hence, their calculations are concerned with Brillouin scattering, while here we are considering Rayleigh scattering, for which accurate experimental information on nonequilibrium fluctuations has been obtained. More recently, Bena et al. have studied the velocity autocorrelation function adopting an hydrodynamic approximation known as Kolmogorov flow [62]; again, due to the nature of the hydrodynamic simplifications performed, the results cannot be compared with those obtained in the present paper.

\subsection{Correlations in the horizontal plane}

A second particular case for which the real-space behavior of the correlations in a liquid subjected to a stationary temperature gradient can be easily studied is in a horizontal plane, that is, in the plane perpendicular to the temperature gradient and far from the boundaries $\left(z=z^{\prime}=L / 2\right)$. We shall analyze here the spatial dependence of the correlations in the horizontal direction near the convective instability using the most-unstable-mode approximation, Eq. (45), which, as was shown in Section 6, yields an excellent representation of the 
structure factor near the convective instability. In this approximation:

$$
G_{\mathrm{NE}}\left(r_{\|}, L / 2, L / 2\right)=\frac{S_{\mathrm{E}} \tilde{S}_{\mathrm{NE}}^{0}}{2 \pi^{2} L^{3}} \int_{0}^{\infty} \frac{\tilde{q}_{\|}^{3} J_{0}\left(\tilde{q}_{\|} \tilde{r}_{\|}\right)}{\left(\tilde{q}_{\|}^{2}+\pi^{2}\right)^{3}-R \tilde{q}_{\|}^{2}} d \tilde{q}_{\|},
$$

where $\tilde{r}_{\|}=r / L$. The integral (52) can be evaluated exactly, when $R<R_{\mathrm{c}}$, but the result is a rather long expression. Instead we prefer to plot in Fig. 6 the resulting $G_{\mathrm{NE}}\left(r_{\|}, z=z^{\prime}=L / 2\right) / G_{\mathrm{NE}}\left(0, z=z^{\prime}=L / 2\right)$ as a function of $r_{\|}$for two values of the Rayleigh number. The solid curve represents the density autocorrelation function for $\epsilon=-0.01$, which is close to the convective instability; the dotted curve represents the same function, but for $\epsilon=-0.7$. For $R$ well below $R_{\mathrm{c}}$, the correlation function is overdamped but, as the value of $R$ approaches $R_{\mathrm{c}}$, it starts to oscillate and the number of oscillations before the correlation function decays to zero increases the closer one approaches $R_{\mathrm{c}}$. To see this mathematically, we have made an asymptotic expansion of $G_{\mathrm{NE}}\left(r_{\|}, z=z^{\prime}=L / 2\right)$ for large $r_{\|}$and, when $R$ is close to $R_{c}$, we obtain:

$$
\begin{aligned}
G_{\mathrm{NE}}\left(r_{\|}, L / 2, L / 2\right) & \simeq \frac{S_{\mathrm{E}} \tilde{S}_{\mathrm{NE}}^{0}}{812^{1 / 4} \pi^{3} L^{3}} \sqrt{\frac{108-151 \epsilon}{-\epsilon \tilde{r}_{\|}}} \exp \left(\frac{-\sqrt{-6 \epsilon}}{4} \pi \tilde{r}_{\|}\right) \\
& \times \cos \left[\frac{\sqrt{2} \pi \tilde{r}_{\|}}{2}-\left(\frac{\pi}{4}-\frac{23 \sqrt{-3 \epsilon}}{36}\right)\right] .
\end{aligned}
$$

The approximation (53) for $\epsilon=-0.01$ is shown in Fig. 6 as a dashed curve, to be compared with the solid curve representing the autocorrelation function as given by Eq. (52) for the same value of $\epsilon$. As can be inferred from Fig. 6, the approximation is excellent for small $\epsilon$ and for large $\tilde{r}_{\|}$. From Eq. (53), the real-space behavior of the autocorrelation function in the horizontal plane as a kind of damped oscillation is evident.

\section{Summary and concluding remarks}

Starting from the linearized fluctuating Boussinesq equations we have derived the structure factor of a horizontal fluid layer taking into account both gravity and finite-size effcts. The resulting expression reproduces the $q^{-4}$ dependence of the nonequilibrium structure factor predicted theoretically [6-8] and confirmed experimentally [10-13] for negative Rayleigh numbers, it accounts for the saturation of the nonequilibrium enhancement of the intensity of the fluctuations at small wave numbers observed by Giglio and coworkers $[22,23,52,53]$ and it is consistent with the experimental observation by Ahlers and coworkers $[28,51]$ of the structure factor close to the convective instability. We have

thus provided a unified approach for describing nonequilibrium fluctuations 
for both negative and positive values of the Rayleigh number, provided that $R<R_{\mathrm{c}}$. Our solution also enables us to evaluate the real-space behavior of the correlation function of the temperature fluctuations which agrees with the results obtained by other investigators for the spatial direction co-incident with the direction of gravity $[14,17,19,60,61]$.

There are still a number of issues that deserve further attention.

First of all, for the sake of mathematical simplicity, we have adopted stressfree boundary conditions in the present paper. For a quantitative comparison with experimental data at very small wave numbers and close to the convective instability modifications due to no-slip boundary conditions should be considered $[25,27,29,31,36]$.

Second, we want to emphasize that neither our solution nor that of Swift and Hohenberg $(\mathrm{SH})$ close to the convective instability yield a Lorentzian wave number dependence on $q$ as is often assumed in the literature since a Lorentzian does not yield the proper asymptotic behavior for either small or large $q$. Moreover, even when the $\mathrm{SH}$ equation is not approximated by a Lorentzian, it does not yield the expected asymptotic $q$ behavior either. We plan to study this issue in more detail in the future.

Finally, while the average local values $\langle\theta\rangle$ and $\langle w\rangle$ are zero, the average cross correlation $\langle\theta \cdot w\rangle$ outside equilibrium is not generally zero. As a result, the coupling between temperature and transverse-velocity fluctuations may cause a small convective heat transfer, even below the instability [29,31]. A satisfactory solution of this effect has not yet been obtained [30-33].

\section{Acknowledgments}

The authors acknowledge some valuable discussions with G. Ahlers, D.S. Cannell, T.R. Kirkpatrick, R. Pérez Cordón and M.G. Velarde. The research at the University of Maryland is supported by the Chemical Sciences, Geosciences and Biosciences Division of the Office of Basic Energy Sciences of the US Department of Energy under Grant No. DE-FG-02-95ER14509. J.V.S. acknowledges the hospitality of the Departamento de Física Aplicada I, Universidad Complutense de Madrid, where the manuscript was completed.

\section{References}

[1] V. Zaitsev, M. Shliomis, Sov. Phys. JETP 32 (1971) 866. 
[2] R. Graham, Phys. Rev. A 10 (1974) 1762.

[3] R. Graham, H. Pleiner, Phys. Fluids 18 (1975) 130.

[4] J. B. Swift, P. C. Hohenberg, Phys. Rev. A 15 (1977) 319.

[5] M. C. Cross, P. C. Hohenberg, Rev. Mod. Phys. 65 (1993) 851.

[6] T. R. Kirkpatrick, E. G. D. Cohen, J. R. Dorfman, Phys. Rev. A 26 (1982) 995.

[7] D. Ronis, I. Procaccia, Phys. Rev. A 26 (1982) 1812.

[8] R. Schmitz, E. G. D. Cohen, J. Stat. Phys. 39 (1985) 285.

[9] B. M. Law, J. V. Sengers, J. Stat. Phys. 57 (1989) 531.

[10] B. M. Law, R. W. Gammon, J. V. Sengers, Phys. Rev. Lett. 60 (1988) 1554.

[11] B. M. Law, P. N. Segrè, R. W. Gammon, J. V. Sengers, Phys. Rev. A 41 (1990) 816.

[12] P. N. Segrè, R. W. Gammon, J. V. Sengers, B. M. Law, Phys. Rev. A 45 (1992) 714.

[13] J. V. Sengers, J. M. Ortiz de Zárate, in: W. Köhler, S. Wiegand (Eds.), Thermal Nonequilibrium Phenomena in Fluid Mixtures, Springer, Berlin, 2001, in press.

[14] A. L. García, M. Malek Mansour, G. C. Lie, E. Clementi, J. Stat. Phys. 47 (1987) 209

[15] J. M. Rubí, A. Díaz-Guilera, L. Torner, Physica A 141 (1987) 220.

[16] C.Z.-W Liu, I. Oppenheim, J. Stat. Phys. 86 (1997) 179.

[17] A. L. García, G. Sonnino, M. Malek Mansour, J. Stat. Phys. 90 (1998) 1489.

[18] M. Malek Mansour, M. Mareschal, G. Sonnino, E. Kestemont, in: M. Mareschal, B. Holian (Eds.), Microscopic Simulations of Complex Hydrodynamic Phenomena, Plenum, London, 1992, p. 87.

[19] A. Suárez, J. P. Boon, P. Grosfils, Phys. Rev. E 54 (1996) 1208.

[20] P. N. Segrè, R. Schmitz, J. V. Sengers, Physica A 195 (1993) 31.

[21] P. N. Segrè, J. V. Sengers, Physica A 198 (1993) 46.

[22] A. Vailati, M. Giglio, Phys. Rev. Lett. 77 (1996) 1484.

[23] A. Vailati, M. Giglio, Prog. Colloid. Polym. Sci. 104 (1997) 76.

[24] J. M. Ortiz de Zárate, R. Pérez Cordón, J. V. Sengers, Physica A 291 (2001) 113.

[25] J. M. Ortiz de Zárate, L. Muñoz Redondo, Euro. Phys. J. B 21 (2001) 135.

[26] T. R. Kirkpatrick, E. G. D. Cohen, J. Stat. Phys. 33 (1983) 639. 
[27] R. Schmitz, E. G. D. Cohen, J. Stat. Phys. 40 (1985) 431.

[28] M. Wu, G. Ahlers, D. S. Cannell, Phys. Rev. Lett. 75 (1995) 1743.

[29] G. Ahlers, M. C. Cross, P. C. Hohenberg, S. Safran, J. Fluid Mech. 110 (1981) 297.

[30] C. W. Meyer, G. Ahlers, D. S. Cannell, Phys. Rev. Lett. 59 (1987) 1577.

[31] H. van Beijeren, E. G. D. Cohen, J. Stat. Phys. 53 (1988) 77.

[32] G. Ahlers, C. W. Meyer, D. S. Cannell, J. Stat. Phys. 54 (1989) 1121.

[33] C. W. Meyer, G. Ahlers, D. S. Cannell, Phys. Rev. A 44 (1991) 2514.

[34] I. Rehberg, S. Rasenat, M. de la Torre Juárez, W. Schöpf, F. Hörner, G. Ahlers, H. R. Brand, Phys. Rev. Lett. 67 (1991) 596.

[35] S. Chandrasekhar, Hydrodynamic and Hydromagnetic Stability, Oxford Univ. Press, Oxford, 1961.

[36] P. C. Hohenberg, J. B. Swift, Phys. Rev. A 46 (1992) 4773.

[37] J. García Ojalvo, A. Hernández Machado, J. M. Sancho, Phys. Rev. Lett. 71 (1993) 1542.

[38] D. D. Gray, A. Giorgini, Int. J. Heat Mass Transfer 19 (1976) 545.

[39] D. J. Tritton, Physical Fluid Dynamics, 2nd Edition, Oxford University Press, Oxford, 1988.

[40] C. Normand, Y. Pomeau, M. G. Velarde, Rev. Mod. Phys. 49 (1977) 581.

[41] F. H. Busse, J. Fluid Mech. 30 (1967) 625.

[42] G. Ahlers, J. Fluid Mech. 98, Part I (1980) 137.

[43] E. Bodenschatz, J. R. de Bruyn, G. Ahlers, D. S. Cannell, Phys. Rev. Lett. 67 (1991) 3078.

[44] L. D. Landau, E. M. Lifshitz, Fluid Mechanics, Addison-Wesley, Reading MA, 1959.

[45] D. Ronis, I. Procaccia, J. Machta, Phys. Rev. A 22 (1980) 714.

[46] B. J. Berne, R. Pecora, Dynamic Light Scattering, Wiley, New York, 1976.

[47] J. V. Sengers, J. M. Ortiz de Zárate, Revista Mexicana de Física (2001), in press.

[48] P. Manneville, Dissipative Structures and Weak Turbulence, Academic Press, San Diego, 1990.

[49] I. S. Gradstein, I. M. Ryzhik, Table of Integrals, Series, and Products, 5th Ed., Academic Press, San Diego, 1994. 
[50] J. R. de Bruyn, E. Bodenschatz, S. W. Morris, S. P. Trainoff, Y. Hu, D. S. Cannell, G. Ahlers, Rev. Sci. Instrum. 67 (1996) 2043.

[51] E. Bodenschatz, W. Pesch, G. Ahlers, Annu. Rev. Fluid Mech. 32 (2000) 709.

[52] A. Vailati, M. Giglio, Nature 390 (1997) 262.

[53] D. Brogioli, A. Vailati, M. Giglio, Phys. Rev. E 61 (2000) R1; J. Phys.: Condens. Matter 12 (2000) A39.

[54] J. García Ojalvo, J. M. Sancho, Noise in Spatially Extended Systems, Springer, New York, 1999.

[55] K. Staliunas, Spatial and temporal spectra of noise driven stripe pattern, available at http://xxx.lanl.gov/abs/cond-mat/0103018 (2001).

[56] W. B. Li, P. N. Segrè, R. W. Gammon, J. V. Sengers, Physica A 204 (1994) 399.

[57] M. A. Scherer, G. Ahlers, F. Hörner, I. Rehberg, Phys. Rev. Lett. 85 (2000) 3754 .

[58] L. Torner, J. M. Rubí, Phys. Rev. A 44 (1991) 1077.

[59] I. Pagonabarraga, J. M. Rubí, L. Torner, Physica A 173 (1991) 111.

[60] H.-P. Breuer, F. Petruccione, Phys. Lett. A 185 (1994) 385.

[61] M. Malek Mansour, J. W. Turner, A. L. García, J. Stat. Phys. 48 (1987) 1157.

[62] I. Bena, M. Malek Mansour, F. Baras, Phys. Rev. E 59 (1999) 5503. 